\title{
35. VOLCANIC ASH FROM CELEBES AND SULU SEA BASINS OFF THE PHILIPPINES (LEG 124): PETROGRAPHY AND GEOCHEMISTRY1
}

\author{
André Pouclet, ${ }^{2}$ Manuel Pubellier, ${ }^{3}$ and Piera Spadea ${ }^{4}$
}

\begin{abstract}
During Leg 124, off the Philippines, volcanic material was recovered in deep-sea sediments dating from the late Oligocene in the Celebes Sea Basin, and from the early Miocene in the Sulu Sea Basin. Chemical and petrological studies of fallout ash deposits are used to characterize volcanic pulses and to determine their possible origin. All of the glass and mineral compositions belong to medium- $\mathrm{K}$ and high- $\mathrm{K}$ calc-alkaline arc-related magmatic suites including high-Al basalts, pyroxene-hornblende andesites, dacites, and rhyolites.

Late Oligocene and early Miocene products may have originated from the Sunda arc or from the SabahZamboanga old Sulu arc. Late early Miocene Sulu Sea tuffs originated from the Cagayan arc, whereas early late Miocene fallout ashes are attributed to the Sulu arc. A complex magmatic production is distinguished in the Plio-Quaternary with three sequences of basic to acidic lava suites. Early Pliocene strata registered an important activity in both Celebes Sea and Sulu Sea areas, from the newly born Sangihe arc (low-alumina andesite series) and from the Sulu, Zamboanga, and Negros arcs (high-alumina basalt series and high-K andesite series). In the late Pliocene and the early Pleistocene, renewal of activity affects the Sangihe-Cotobato arc as well as the Sulu and Negros arcs (same magmatic distinctions). The last volcanic pulse took place in the late Pleistocene with revival of all the present arc systems.
\end{abstract}

\section{INTRODUCTION}

Volcaniclastic material was recovered in Oligocene to Quaternary sediments of the Celebes Sea and Sulu Sea Basins during Leg 124 (Fig. 1). It consists of dispersed glass shards and magmatic minerals, discrete ash layers, and reworked pyroclastic and hyaloclastic tuffs (nomenclature. grade size limits and terms according to Fisher, 1961, 1966, pers. comm., and Fisher and Schmincke, 1984). The tephrostratigraphic record relates the history of volcanic activity surrounding the basins: north Sulawesi and Sangihe arcs, old and present Philippines arc systems (Manila, Cotobato, and Sulu arcs). We examine the petrographic and chemical compositions of this volcaniclastic material, mainly related to explosive activity, in an attempt to determine their magmatic origin and evolution.

\section{ASH AT LEG 124 SITES}

Two sites were drilled in the Celebes Sea Basin (767 and 770 ) and three in the Sulu Sea Basin (768, 769, and 771). The tephrachronologic investigations are presented in Pubellier et al., (this volume).

\section{Celebes Sea}

Sites 767 and 770 are located in the northeastern Celebes Sea. Numerous volcanoes surround the sites at distances of 200 to $400 \mathrm{~km}$. They are located in the North Sulawesi arc, Sangihe arc, central Mindanao and Cotobato arcs, and Sulu ridge.

Important Plinian activity of rhyolitic composition is registered from 2.5 Ma to the Holocene. A second pulse of

\footnotetext{
${ }^{1}$ Rangin, C.. Silver. E. A., von Breymann, M. T., et al., 1990. Proc. ODP, Sci. Results, 124:College Station, TX (Ocean Drilling Program).

${ }^{2}$ Laboratoire de Géotectonique et Pétrologie, Université d'Orléans. 45067 Orleans, France.

${ }^{3}$ Laboratoire de Géotectonique, Université Pierre et Marie Curie. 75252 Paris. France.

${ }^{4}$ Istituto di Scienze della Terra, Universita di Udine, Italy.
}

volcanism occurred between 8 and $4 \mathrm{Ma}$ and is characterized by more mafic products (dacite and andesite). The Miocene strata, poor in glass fragments, include some altered andesitic microscoria and pyroclastic minerals (feldspars, pyroxenes, and amphiboles). Variably altered andesitic-to-rhyolitic glasses occur within Oligocene sediments. They are abundant in early Oligocene beds and dispersed in late Oligocene beds, indicating distant activity.

\section{Sulu Sea}

Site 768 is located in the southeastern part of the Sulu Sea. Site 769 is in the southeast flank of the Cagayan Ridge and Site 771 is on the Cagayan Ridge. Active and Quaternary volcanoes are very near these sites, in the Cagayan Ridge, and 150 to $300 \mathrm{~km}$-distant, in the Sulu Ridge, and on the Panay and Negros Islands.

Sites 768 and 769 registered volcanic activity since late early Miocene time. Site 771 was devoted to basement objectives. Numerous dacitic to rhyolitic ash layers are interbedded in late Miocene to Holocene sediments. They indicate important proximal explosive activity. More mafic and andesitic products are encountered in early Pleistocene beds. Late early to middle upper Miocene strata are poor in volcanic material, which consists of dispersed rhyolitic glass shards, magmatic minerals, and scarce andesitic to dacitic altered glass fragments. Important activity took place in the late early Miocene with the deposition of thick vitric and lithic tuff and lapillistone of pyroclastic (Site 768) or hyaloclastic (Site 769) origin. These tuffs overlie the lava flows of the basement from which they are separated only by a few meters of brown clay.

\section{PETROCHEMICAL STUDY}

\section{Sampling}

A total of 70 samples were selected to obtain a representative chronological record of the magmatic characteristics in glass and mineral composition of the different areas. Table 1 gives the location and the significant petrographical, chemical, and mineralogical features of the samples. Volcaniclastic 


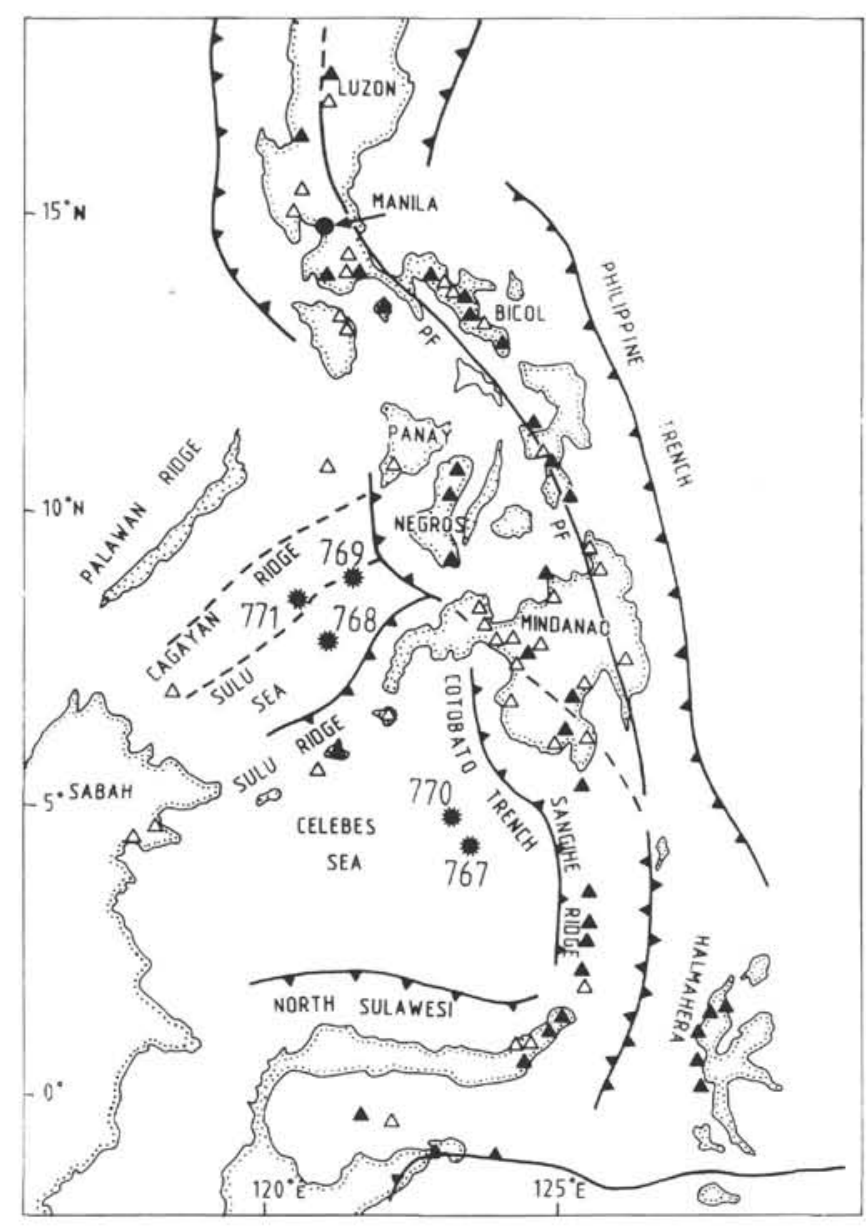

Figure 1. Geographic and structural setting of Leg 124. Sites 767, 768, 769, 770, and 771. PF, Philippine Fault. Full triangles, Holocene volcanoes; open triangles, Pliocene-Pleistocene volcanoes.

material corresponds to vitric ashes and to magmatic minerals; it was investigated in 26 ash layers (glass $>60 \%$ ), 12 ash-bearing beds $(10 \%<$ glass $<60 \%), 4$ tuffs, and 28 crystal-rich beds. This selected material is from fallout deposits.

Chronostratigraphic determinations give the following age groups from present to old: 1, Holocene-late Pleistocene; 2, middle Pleistocene; 3 , early Pleistocene: 4, late Pliocene; 5, early Pliocene; 6 , late Miocene; 7 , middle Miocene; 8 , early Miocene: 9 , late Oligocene; 10 , early Oligocene.

We observe three kinds of ash deposits:

1. H (homogeneous), discrete ash layers from single eruptions with weak mixing, good sorting and single mineral and chemical composition. That corresponds to type I of Huang (1980).

2. T (heterogeneous), mixed ashes from frequent, closespaced eruptions and/or synchronous eruptions from multiple chambers (magma mingling). That is type II of Huang (1980).

3. D, dispersed ashes in sediments.

The shape of glass fragments is given in order of abundance. Sizes and morphologies of fragments indicate Plinian eruptions (shards, tubular micropumices, bubble-wall, or $\mathrm{U}$ and Y-reticulite fragments), or sub-Plinian to Vulcanian eruptions (shards and microscorias).

\section{Analytical Method}

We used an electron-probe microanalyzer (EPMA) (Camebax C-U-B, Orleans, France), at an accelerating voltage of 15 $\mathrm{kV}$, a specimen current of $5 \mathrm{nA}$, a beam diameter of 1 to $5 \mu \mathrm{m}$, and a time count of $10 \mathrm{~s}$. Specimen current and time count are as low as possible for spot analyses of glass. A large defocused beam is generally required for that kind of material, but it does not allow one to analyze tiny fragments or heterogeneous glasses. Frequently, the width of shards and reticulite fragments is less than $10 \mu \mathrm{m}$, and glasses contain microcrysts of tridymite, feldspar, and oxides.

The analytical conditions bring about $\mathrm{Na}$ and $\mathrm{K}$ evaporation, a loss (shifting) of $\mathrm{Al}$ and some other elements, and, consequently, an Si-enrichment. Measurements were corrected by systematic adjusting with reference to high-quality synthetic glasses of known composition.

\section{Significance of Glass Analyses}

The total amount of oxide components range from $86 \%$ to $98 \%$. The deficit comes from the initial volatile component, and from hydration and post-depositional alteration in seawater. The initial volatile component (magmatic volatile content and contamination during volcanic processes) may be as high as $5 \%$ in dacitic to rhyolitic glasses. Concurrently, hydration occurs rapidly to reach a saturation point of $4 \%$ to $5 \%$. Beyond this point, secondary clay minerals develop in the glass giving an optically visible alteration (Pouclet et al. 1985, 1990).

To test the possible effect of hydration on the chemical composition, we calculated the correlation coefficients between oxides content and the analytical deficit (total of oxides) in all the felsic glass analyses (when no alteration was detected). There is a negative correlation for $\mathrm{SiO}_{2}(-0.689)$. But positive correlations affect, in decreasing order, $\mathrm{Na}_{2} \mathrm{O}$ $(+0.846), \mathrm{Al}_{2} \mathrm{O}_{3}(+0.790), \mathrm{Fe}_{2} \mathrm{O}_{3}$ total and $\mathrm{MnO}(+0.773), \mathrm{TiO}_{2}$ $(+0.758), \mathrm{MgO}(+0.711), \mathrm{CaO}(+0.688)$, and $\mathrm{K}_{2} \mathrm{O}(+0.517)$. This is due to the relative leaching of these elements during hydration.

Then we calculated, for the whole population, the correlation coefficients between $\mathrm{SiO}_{2}$ and the oxides: $\mathrm{TiO}_{2},-0.843$; $\mathrm{Al}_{2} \mathrm{O}_{3},-0.858 ; \mathrm{Fe}_{2} \mathrm{O}_{3}$ total and $\mathrm{MnO},-0.880 ; \mathrm{MgO},-0.892$; $\mathrm{CaO},-0.931 ; \mathrm{Na}_{2} \mathrm{O},-0.439 ; \mathrm{K}_{2} \mathrm{O},-0.154$. All the oxides except $\mathrm{K}_{2} \mathrm{O}$ and, to a lesser extent, $\mathrm{Na}_{2} \mathrm{O}$, strongly decrease as $\mathrm{SiO}_{2}$ increases, from basic to acidic composition (differentiation effect). Taking into account this effect and the hydration effect, we corrected the raw data by adjusting the $\mathrm{SiO}_{2}$ values, compared to the other elements, and we calculated the analyses to $100 \%$ (water-free) to obtain an internally consistent analytical population (Table 2). However, in the case of high hydration $(7 \%-9 \%)$ and possible smectite formation, the correction is inadequate. Important alkali loss is the cause of high normative corundum content (late to middle Miocene glasses of Site 768).

\section{Chemical Composition of Glasses}

\section{Analytical Data}

Volcanic glass was analyzed in 45 samples (Table 1). Usually, an average of 6 to 12 analyses per sample (range, 1 to 20 analyses) is done, depending the amount, homogeneity, and freshness of vitric fragments. In addition, approximate compositions were estimated in 7 samples for scarce and highly altered glass fragments. Silica content varies from $53 \%$ to $79 \%$. Glasses are only acidic $\left(\mathrm{SiO}_{2}>68 \%\right)$ in 31 samples, and only basic or basic to intermediate $\left(53 \%<\mathrm{SiO}_{2}<68 \%\right)$ in 12 samples: they are both basic and acidic in 2 samples. Most of the ash layers are homogeneous. 
Normative compositions are strongly oversaturated $(20<$ quartz $<40)$ and aluminous $(1<$ corundum $<3)$ for acidic glasses, oversaturated $(4<$ quartz $<20)$ for intermediate glasses. and saturated $(0<$ quartz $<4)$ to undersaturated $(0<$ olivine $<8$ ) for basic glasses, some of them having normative nepheline. Very high normative corundum-rich glasses are discarded. Oversaturated mafic compositions are encountered in all the sites. Undersaturated mafic compositions characterize the Sulu and Cagayan Sites. Table 2 presents a selection of analyses, in chronologic order, for the three areas: Celebes, Sulu, and Cagayan.

On-board major- and minor-element X-ray fluorescence (XRF) analyses were performed on 16 volcaniclastic samples (Rangin, Silver, von Breymann, et al., 1990). Analyses of raw or incompletely washed samples concern fresh or altered vitric ashes, fallout, and detrital or authigenic minerals, as well as biogenic material. Because of an insufficient amount of material, few samples were purified. Consequently, uncertainty remains about magmatic compositions. There are discrepancies between microprobe glass analyses (EPMA) and whole-sample XRF analyses. For dacite ashes, XRF measurements give lower Si-content and higher $\mathrm{Fe}, \mathrm{Mg}, \mathrm{Ca}$, and $\mathrm{V}$-contents, possibly due to the presence of plagioclase, pyroxene, and/or amphibole in the raw samples. For rhyolite ashes, XRF measurements give higher $\mathrm{Al}, \mathrm{Fe}, \mathrm{Mg}, \mathrm{Ca}, \mathrm{K}$, and $\mathrm{Ba}$-contents, possibly due to the presence of pyroxene, biotite, and alkaline feldspar. Only one ash was sufficiently clean for analysis of rare-earth elements (REE) by emission spectrometry ICP method: Sample 7 (124$767 \mathrm{~B}-3 \mathrm{H}-1,88-90 \mathrm{~cm}$ ). This is a medium-K rhyolite from mid-Pleistocene strata (Table 3). Low REE abundances and slight light REE-enrichment (chondrite normalized $\mathrm{La} / \mathrm{Yb}=$ 2.80 ) are consistent with evolved products from island-arc suites. A moderately negative Eu anomaly may have resulted from feldspar fractionation.

\section{Variation Diagrams}

Analyses were plotted on $\mathrm{SiO}_{2}$ vs. oxides variation diagrams (Fig. 2). $\mathrm{TiO}_{2}$, total $\mathrm{Fe}_{2} \mathrm{O}_{3}, \mathrm{MgO}$, and $\mathrm{CaO}$ decrease with increasing $\mathrm{SiO}_{2} . \mathrm{Al}_{2} \mathrm{O}_{3}$ shows no variations, or weakly increases from $53 \%$ to $68 \% \mathrm{SiO}_{2}$ and then decreases. In the same silica range, alkalies increase and then decrease. Important chemical variations affect mafic and intermediate glass analyses, especially for the alumina and alkalies content. This is partly due to heterogeneity of glasses including numerous feldspar microcrysts. However, taking into account the averaged calculations, mafic glasses are classified into two groups: Al-rich group $\left(\mathrm{Al}_{2} \mathrm{O}_{3}>16 \%\right)$, occurring at all the sites, and Al-poor group $\left(\mathrm{Al}_{2} \mathrm{O}_{3}<16 \%\right)$, at Sites 767 and 769 . On the $\mathrm{Na}_{2} \mathrm{O}-\mathrm{K}_{2} \mathrm{O}-\mathrm{CaO}$ diagram (Fig. 3), the alkali composition is sodic; but some Celebes and Sulu seas volcanics show a potassic trend.

As a whole, considering the $\mathrm{Al}, \mathrm{Na}, \mathrm{K}$, and $\mathrm{Ti}$ contents and the lack of Fe-enrichment (A-F-M diagram, Fig. 4), chemical compositions of glasses may be related to moreor-less potassic calc-alkaline magmatic series, For the $\mathrm{SiO}_{2}$ $\mathrm{K}_{2} \mathrm{O}$ diagram (Fig. 2), compositions belong to the mediumpotassic andesite series and to the high-potassic andesite (shoshonite) series.

\section{Chemical Composition of Minerals}

Pyroclastic crystals and epiclastic detrital grains are generally present in selected samples. They consist of feldspar, pyroxene, amphibole, oxides, biotite, and rare olivine. They have been analyzed when not too altered (Table 4). Table 1 presents summarized data.

\section{Feldspars}

Feldspars were found and analyzed in almost all the samples. They exhibit a very large compositional range, from An 93 to An 12 for plagioclases, and to Or 98 for alkaline feldspars. Zonation is rarely observed because fragments are small (e.g., An 76-60 in a crystal from Sample 124-767B$28 \mathrm{X}-1,135-138 \mathrm{~cm}$ ). Associated fragments in a layer, however, commonly show various An-contents and plagioclasealkaline feldspar pairs. There is a correlation between feldspar An and Or-content and glass petrochemical types: An 93-60 with basaltic andesite, An 75-40 with andesite, An 50-20 and Or 20-60 with dacite, An 40-12 and Or 40-98 with rhyolite. Acidic ash beds may include An-rich plagioclase clasts or detrital grains attributed to magmatic xenocrysts or to erosional products of volcanic terrane.

There are different crystallization trends on the An-Ab-Or diagram (Fig. 5), particularly in the intermediate oligoclaseanorthoclase compositional area. Extent of K-rich plagioclase and of Ca-rich alkaline feldspar solid solutions indicates high $\left(900^{\circ} \mathrm{C}\right)$ to low $\left(650^{\circ} \mathrm{C}\right)$ temperature of crystallization of intermediate evolved liquids (Barth, 1962; Seck, 1971). There is no evident correlation between $\mathrm{Al}$ and $\mathrm{K}$-chemical characters of glasses and the temperature crystallization trends of feldspars. The latter may be controlled first by the magmato-tectonic setting of volcanoes. Coexisting plagioclases and alkaline feldspars have been found in polymineral fragments, for example in Site 767:

1, An60 - Or1.8 and An12 - Or24 (Sample 124-767B-28X-1, $135-138 \mathrm{~cm})$;

2, An53 - Or1.6 and An11 - Or55 (Sample 124-767B-7H-4, $71-73 \mathrm{~cm})$

3, An37 - Or3.1 and An1.8 - Or68 (Sample 124-767B-76X-4, $110-112 \mathrm{~cm})$;

4, An24 - Or3.1 and An1.6 - Or44 (Sample 124-767C-6R-1, $17-19 \mathrm{~cm})$.

They correspond to high-temperature crystallization trends (associations 1 and 2, andesitic-dacitic liquid), and to low-temperature crystallization trends (associations 3 and 4, dacitic-rhyolitic liquid).

\section{Pyroxenes}

Clinopyroxenes (CPX) and orthopyroxenes (OPX) have been analyzed in 17 samples. CPXs are determined as diopside: En 49-43, Wo 46-37 (Fig. 6). Except for two Al and $\mathrm{Fe}^{3+}$-rich pyroxenes from basaltic andesite ash layers (Samples 124-767B-2H-7, 36-38 cm, and 124-767B-21X-5, 139$141 \mathrm{~cm}$ ), they have high $\mathrm{Si}$ and low $\mathrm{Al}, \mathrm{Ti}$, and $\mathrm{Na}$-contents indicating sub-alkaline characters: $\mathrm{SiO}_{2} 50 \%, \mathrm{Al}_{2} \mathrm{O}_{3}<4 \%$, $\mathrm{Al}_{\text {IV }}<0.10$, and $\mathrm{Ti}<0.05$ for six oxygens. They are associated with mafic glasses. Rare detrital grains occur in rhyolitic beds.

OPXs are bronzite to hypersthene: En 77-51, Wo 0.2-7.0. They are associated with acidic glasses and indicate a calcalkaline signature,

In a lithic fragment from Sample 124-769B-3H-6, 25-27 $\mathrm{cm}$, two pyroxenes coexist with plagioclase An48 and andesitic glass. A CPX-OPX geothermometer (Wells, 1977) indicates $1042^{\circ} \mathrm{C}$. This liquidus temperature assigned to plagioclase-liquid equilibrium (Kudo and Weill, 1970; Mathez, 1973) corresponds to an $\mathrm{H}_{2} \mathrm{O}$ pressure of $2 \mathrm{kbars}$, in good agreement with the thermobarometric pattern of an andesitic magma. 
Table 1. Leg 124 core location and petrographic, chemical, and mineralogical features of the tephra samples. Age group and type, see text.

\begin{tabular}{|c|c|c|c|c|c|c|c|c|c|c|c|c|c|}
\hline $\begin{array}{c}\text { Sample } \\
\text { no. }\end{array}$ & $\begin{array}{l}\text { Core, section, } \\
\text { interval }(\mathrm{cm})\end{array}$ & $\begin{array}{c}\text { Age } \\
\text { group }\end{array}$ & $\begin{array}{l}\text { Lithology \% } \\
\text { sand-silt- } \\
\text { clay }\end{array}$ & $\underset{\%}{\text { Glass }}$ & $\begin{array}{l}\text { Ash } \\
\text { type }\end{array}$ & type & $\begin{array}{l}\text { Volcanic glass } \\
\text { medium size range } \\
\text { (extra range) }\end{array}$ & $\mathrm{SiO}_{2}$ range & $\begin{array}{l}\text { Alk. feldsp: } \\
\text { An/Or rang }\end{array}$ & $\begin{array}{l}\text { Miner } \\
\text { Plagioclase } \\
\text { An/Or range }\end{array}$ & $\begin{array}{c}\text { Pyroxene } \\
\text { En/Wo range }\end{array}$ & $\begin{array}{c}\text { Amphibole } \\
\text { name }\end{array}$ & others \& notes \\
\hline & $770 \mathrm{~B}$ & & & & & & & & & & & & \\
\hline 1 & $1 \mathrm{R}-2,19-21$ & 1 & $70-30-0$ & 65 & $\mathrm{~T}$ & sh, bw & $50-150(200)$ & $77.07-77.95$ & & $42-38 / 1.2-1.6$ & & $\mathrm{Mg}-\mathrm{Hb}$ & \\
\hline 2 & $\begin{array}{l}\text { 1R-3, 75-77 } \\
767 \mathrm{~A}\end{array}$ & 1 & $80-20-0$ & 60 & $\mathrm{H}$ & sh, bw & $50-150$ & $77.66-78.13$ & & $36 / 2.0$ & & $\mathrm{Mg}-\mathrm{Hb}$ & \\
\hline \multirow[t]{2}{*}{4} & $\begin{array}{l}1 \mathrm{H}-1,74-76 \\
1 \mathrm{H}-3,68-70\end{array}$ & $\begin{array}{l}1 \\
1\end{array}$ & $\begin{array}{l}20-80-0 \\
90-10-0\end{array}$ & $\begin{array}{l}30 \\
90\end{array}$ & $\begin{array}{l}\mathrm{D} \\
\mathrm{T}\end{array}$ & $\begin{array}{l}\text { sh } \\
\text { sh, bw }\end{array}$ & $\begin{array}{l}30-80 \\
60-250(400)\end{array}$ & $\begin{array}{l}77.76-79.63 \\
73.80-75.33\end{array}$ & & $34 / 2.0$ & & $\mathrm{Mg}-\mathrm{Hb}$ & \\
\hline & $767 \mathrm{~B}$ & & & & & & & & & & & & \\
\hline 5 & $1 \mathrm{H}-3,77-79$ & 1 & $60-40-0$ & 85 & G & sh, bw, mp & $30-250(350)$ & $73.06-76.15$ & & $83-60 / 0.4-1.4$ & $46-43 / 39-37$ & $\mathrm{Mg}-\mathrm{Hb}$ & Ol (Fo 79.5) \\
\hline $\begin{array}{l}6 \\
7\end{array}$ & $\begin{array}{l}2 \mathrm{H}-7,36-38 \\
3 \mathrm{H}-1,88-90\end{array}$ & $\begin{array}{l}2 \\
2\end{array}$ & $\begin{array}{l}80-20-0 \\
80-20-0\end{array}$ & $\begin{array}{l}40 \\
90\end{array}$ & $\begin{array}{l}\mathrm{T} \\
\mathrm{H}\end{array}$ & $\begin{array}{l}\text { bw, sh, mp } \\
\text { bw, sh, mp }\end{array}$ & $\begin{array}{l}50-150 \\
60-200\end{array}$ & $\begin{array}{l}58.49-67.13 \\
75.63-76.56\end{array}$ & & $21 / 11$ & $40 / 41$ & & \\
\hline 8 & $7 \mathrm{H}-4,71-73$ & 3 & $80-20-0$ & 90 & $\mathrm{H}$ & sh, bw & $60-200$ & $74.12-77.51$ & $11 / 55$ & $53-35 / 1.6-4.3$ & $66-65 / 2.7-2.2$ & $\mathrm{Mb}-\mathrm{Hb}$ & Mt (6.5-9.5) \\
\hline 9 & $8 \mathrm{H}-4,21-23$ & 3 & $80-20-0$ & 65 & $\mathrm{~T}$ & bw, sh, mp & $40-120(600)$ & $77.11-78.32$ & $0 / 98$ & $35-30 / 0.7-1.3$ & $66-63 / 2.1-2.3$ & $\mathrm{Mg}-\mathrm{Hb}$ & Ep \\
\hline 10 & $9 \mathrm{H}-3,85-87$ & 3 & $70-30-0$ & 65 & $\mathrm{~T}$ & $\mathrm{bw}, \mathrm{sh}, \mathrm{mp}$ & $40-110(250)$ & $77.09-79.14$ & $5 / 45$ & $37-30 / 0.7-1.1$ & $64 / 1.9$ & $\mathrm{Mg}-\mathrm{Hb}$ & \\
\hline 11 & $10 \mathrm{H}-6,83-85$ & 3 & $70-30-0$ & 85 & $\mathrm{H}$ & $\mathrm{sh}, \mathrm{bw}, \mathrm{mp}$ & $30-120(180)$ & $77.97-79.15$ & & $42-31 / 2.3-3.2$ & $70 / 0.2$ & & \\
\hline 12 & 10X-CC, $12-14$ & 3 & $10-60-30$ & 60 & $\mathrm{H}$ & sh, bw & $25-40(100)$ & $75.81-77.06$ & & & & & \\
\hline 13 & $21 X-5,139-141$ & $4-5$ & $20-80-0$ & 60 & $\mathrm{~T}$ & sh & $30-50(180)$ & $59.09-64.54$ & $4 / 41$ & $93-75 / 0.3-1.0$ & $49-45 / 43-41$ & $\mathrm{P}-\mathrm{Hb}$ & alt. Mt \\
\hline 14 & $24 \mathrm{X}-3,90-92$ & 5 & $30-70-0$ & 15 & D & sh, mp & $30-80(160)$ & $59.56-71.27$ & & $73-50 / 1.0-2.6$ & & $\mathrm{P}-\mathrm{Hb}$ & \\
\hline 15 & $26 \mathrm{X}-5,57-59$ & 5 & $70-30-10$ & 80 & $\mathrm{~T}$ & bw, sh & $50-120$ & 76.96-78.01 & & $34-31 / 2.4-3.0$ & & & alt. Mt \\
\hline 16 & $28 \mathrm{X}-1,135-138$ & 5 & $40-60-0$ & $\operatorname{tr}$ & D & gr & $30-50$ & (61) & $12-0 / 24-90$ & $71-22 / 1.3-9.2$ & $44 / 40$ & & Mt (8.8-9.2), smect \\
\hline 17 & $30 X-3,54-56$ & 5 & $90-10-0$ & $\mathrm{n}$ & & & & & $0.3 / 71$ & $23 / 5.1-5.3$ & & $\mathrm{Mg}-\mathrm{Hb}$ & $\mathrm{Bi}(61-58 ; 3.9-3.5)$ \\
\hline 18 & $32 \times-3,97-99$ & 5 & $40-30-30$ & tr & D & $\mathrm{gr}$ & $30-50$ & (59) & & $47-36 / 1.0-2.2$ & & & \\
\hline 19 & $40 \mathrm{X}-1,18-20$ & 6 & $20-40-40$ & $\mathrm{n}$ & & & & & $1.2 / 74$ & $42-40 / 2.4-2.6$ & & $\mathrm{Mg}-\mathrm{Hb}$ & \\
\hline 20 & $43 X-2,21-23$ & 6 & $10-40-50$ & $\mathrm{n}$ & & & & & & $45 / 1.2$ & & & \\
\hline 21 & $60 \times-7,19-20$ & 7 & $0-40-60$ & $\mathrm{n}$ & & & & & $0 / 85$ & $11 / 4.4$ & & & \\
\hline 22 & $67 X-5,17-18$ & $7-8$ & $10-70-20$ & $\mathrm{n}$ & & & & & $0 / 86$ & $80 / 0.9$ & & & $\mathrm{Qtz}, \mathrm{Ab}$ \\
\hline 23 & $73 X-7,22-24$ & 8 & $0-10-90$ & $\mathrm{n}$ & & & & & & $46 / 1.3$ & & & Illite \\
\hline \multirow[t]{2}{*}{24} & $76 X-4,110-112$ & $8-9$ & $30-60-10$ & $\mathrm{n}$ & & & & & $2-0 / 68-97$ & $37-13 / 0.8-6.1$ & & & Qtz \\
\hline & $767 \mathrm{C}$ & & & & & & & & & & & & \\
\hline 25 & $6 \mathrm{R}-1,17-19$ & 9 & $50-40-10$ & tr & D & gr & $80-150$ & 77.58 & $2-0 / 44-97$ & $46-24 / 2.2-3.1$ & & & Mt (9.5), Ilm (28.8) \\
\hline 26 & $6 \mathrm{R}-2,147-149$ & 9 & $10-80-10$ & tr & D & gr & $20-100$ & (62) & $0.7 / 70$ & & & & Mt (11.2), alt. glass \\
\hline 27 & 7R-CC, $8-10$ & 10 & $10-80-10$ & $\mathrm{n}$ & & & & & $0.7 / 70$ & & & & \\
\hline \multirow[t]{2}{*}{28} & $8 \mathrm{R}-1,136-138$ & 10 & $30-70-10$ & 30 & D & gr & $10-130$ & $75.84-76.79$ & & $53-42 / 0.9-4.0$ & & & Mt (15.9), alt. glass \\
\hline & $768 \mathrm{~B}$ & & & & & & & & & & & & \\
\hline 29 & $1 \mathrm{H}-2,99-101$ & 1 & $10-80-10$ & $\mathrm{n}$ & & & & & & $11-8 / 24-30$ & & & \\
\hline 30 & $2 \mathrm{H}-1,41-43$ & 1 & $80-20-0$ & 70 & $\mathrm{~T}$ & sh, bw & $50-200(500)$ & $77.15-77.74$ & & & & & \\
\hline 31 & $3 \mathrm{H}-1,54-56$ & $1-2$ & $50-40-10$ & 10 & D & sh & $15-80(120)$ & $77.57-77.93$ & $0 / 79$ & $34 / 2.0$ & & & $\mathrm{Bi}(66-63 ; 3.4-3.1)$ \\
\hline 32 & $3 \mathrm{H}-3,118-121$ & $1-2$ & $60-40-0$ & 60 & $\mathrm{H}$ & sh, bw & $30-100$ & $78.11-78.58$ & $12 / 31$ & $87-41 / 0-1.2$ & & $\mathrm{Mg}-\mathrm{Hb}$ & \\
\hline 33 & $4 \mathrm{H}-2,148-150$ & 2 & $60-40-0$ & 60 & $\mathrm{H}$ & bw, sh, mp & $40-110$ & $76.14-77-72$ & & $40-36 / 1.9-2.0$ & & $\mathrm{Mg}-\mathrm{Hb}$ & Mt (5.4) \\
\hline 34 & $4 \mathrm{H}-6,65-67$ & 2 & $70-30-0$ & 80 & $\mathrm{~T}$ & $\mathrm{sc}$ & $40-120(200)$ & $56.55-62.11$ & & $88-53 / 0.3-3.8$ & $43 / 44$ & & Mt (7.1) \\
\hline 35 & $4 \mathrm{H}-7,45-47$ & 2 & $80-20-0$ & 85 & $\mathrm{~T}$ & bw, sh, mp & $50-200(300)$ & $57.40-67.53$ & & $20-14 / 11-20$ & & & \\
\hline 36 & $4 \mathrm{H}-7,82-84$ & 2 & $80-20-0$ & 80 & $\mathrm{H}$ & sc & $50-120$ & $54.51-57.75$ & & $85 / 0.9$ & $42 / 43$ & & Mt (7.6) \\
\hline 37 & $12 \mathrm{H}-7,22-24$ & 3 & $10-30-60$ & $\mathrm{n}$ & & & & & $1 / 24$ & $67-39 / 0.4-3.0$ & & Fe-P-Hb & $\mathrm{Bi}(71 / 3.2)$ \\
\hline 38 & $14 \mathrm{H}-3,113-115$ & $3-4$ & $0-20-80$ & 5 & D & $\mathrm{gr}, \mathrm{sh}$ & $30-50$ & $77.62-78.07$ & & & & $\mathrm{Mg}-\mathrm{Hb}$ & \\
\hline 39 & $15 \mathrm{H}-2,29-31$ & 4 & $60-40-0$ & 8 & D & sh, .g & $50-200$ & $73.60-74.36$ & $10 / 38$ & $48-44 / 0.5-0.9$ & & $\mathrm{Mg}-\mathrm{Hb}$ & \\
\hline 40 & $16 \mathrm{H}-2,49-51$ & 4 & $10-30-60$ & $\mathrm{n}$ & & & & & & $38-15 / 0.9-8$ & & & Mt (4.6), Ilm (26.5) \\
\hline 41 & $16 \mathrm{H}-3,118-120$ & 4 & $50-50-0$ & tr & D & gr & $30-80$ & 79.25 & $6 / 42$ & $33-27 / 1.8-2.8$ & & $\mathrm{Mg}-\mathrm{Hb}$ & alt. $\mathrm{Bi}$ \\
\hline 42 & $18 \mathrm{H}-4,106-108$ & 5 & $50-50-0$ & 10 & D & sh, gr & $30-100$ & (63) & $8 / 56$ & $42 / 2.5$ & & & alt. glass \\
\hline 43 & $22 \mathrm{H}-1,72-73$ & $5-6$ & $60-30-10$ & tr & D & $\mathrm{gr}, \mathrm{sh}$ & $40-100$ & $78.01-78.58$ & & $53-34 / 0.5-2.9$ & & $\mathrm{Mg}-\mathrm{Hb}$ & alt. glass \\
\hline 44 & $22 \mathrm{H}-5,104-105$ & $5-6$ & $30-40-30$ & $\mathrm{n}$ & & & & & $.6-0.2 / 59-83$ & $41-29 / 3.5-7.5$ & & & $\operatorname{llm}(41.7)$ \\
\hline 45 & $23 \mathrm{H}-3,101-102$ & 6 & $30-50-20$ & tr & D & sh, g & $50-100$ & $77.45-77.99$ & & $40-39 / 1.0-1.3$ & $65 / 3.4$ & $\mathrm{Mb}-\mathrm{Hb}$ & alt. glass \\
\hline 46 & $28 \mathrm{X}-3,91-93$ & 6 & $60-40-0$ & $\mathrm{n}$ & & & & & & $42-39 / 1.0-1.3$ & & $\mathrm{Mg}-\mathrm{Hb}$ & \\
\hline
\end{tabular}


Table 1 (continued).

\begin{tabular}{|c|c|c|c|c|c|c|c|c|c|c|c|c|c|}
\hline $\begin{array}{c}\text { Sample } \\
\text { no. }\end{array}$ & $\begin{array}{l}\text { Core, section, } \\
\text { interval }(\mathrm{cm})\end{array}$ & $\begin{array}{l}\text { Age } \\
\text { group }\end{array}$ & $\begin{array}{l}\text { Lithology \% } \\
\text { sand-silt- } \\
\text { clay }\end{array}$ & $\underset{\%}{\text { Glass }}$ & $\begin{array}{l}\text { Ash } \\
\text { type }\end{array}$ & type & $\begin{array}{l}\text { Volcanic glass } \\
\text { medium size range } \\
\text { (extra range) }\end{array}$ & $\mathrm{SiO}_{2}$ range & $\begin{array}{l}\text { Alk. feldspar } \\
\text { An/Or range }\end{array}$ & $\begin{array}{l}\quad \text { Minera } \\
\text { Plagioclase } \\
\text { An/Or range }\end{array}$ & $\begin{array}{l}\text { Pyroxene } \\
\text { En/Wo range }\end{array}$ & $\begin{array}{c}\text { Amphibole } \\
\text { name }\end{array}$ & others \& notes \\
\hline 47 & $29 X-4,49-51$ & 6 & $10-30-60$ & tr & D & sh & $80-90(300)$ & $77.68-78.81$ & $5 / 60$ & $51-45 / 0.5-2.4$ & & $\mathrm{Mg}-\mathrm{Hb}$ & alt. glass \\
\hline \multirow[t]{2}{*}{48} & $35 \mathrm{X}-1,117-119$ & 6 & $20-30-50$ & $\mathrm{n}$ & & & & & $0 / 97$ & $54-20 / 0.7-6.6$ & & $\begin{array}{l}\text { Ts-Mg- } \\
\mathrm{Hb}\end{array}$ & \\
\hline & $768 \mathrm{C}$ & & & & & & & & & & & & \\
\hline 49 & $31 \mathrm{R}-4,61-63$ & 7 & $5-30-65$ & tr & D & gr & $20-70$ & 77.61 & $0.1 / 96$ & $62 / 1.8$ & & & \\
\hline 50 & $32 \mathrm{R}-1,144-146$ & 7 & $5-30-65$ & tr & $\mathrm{T}$ & sh, bw, mp & $80-160(250)$ & $77.87-78.64$ & & $43-39 / 2.8-3.9$ & $65-51 / 2-1.4$ & & Qtz, Ilm (47.3) \\
\hline 51 & $32 \mathrm{R}-2,61-63$ & 7 & $70-30-0$ & 95 & $\mathrm{~T}$ & sh, bw & $50-250(300)$ & $76.25-78.80$ & & $50 / 2$ & & & \\
\hline 52 & $39 \mathrm{R}-3,62-65$ & 7 & $20-70-10$ & 10 & D & $\mathrm{gr}, \mathrm{sh}$ & $20-70$ & 67.95 & $0 / 94$ & & & & alt. glass \\
\hline 53 & $39 \mathrm{R}-4,72-74$ & 7 & $20-70-10$ & 15 & D & $\mathrm{gr}, \mathrm{sh}$ & $25-100$ & $73.51-74.73$ & $0.4 / 79$ & $64-46 / 1.5-2.2$ & & & alt. $\mathrm{Bi}$ \\
\hline 54 & $52 R-4,133-135$ & 8 & & 70 & tuff & & & & $0.2 / 90$ & $80-35 / 0.6-5.9$ & $46-43 / 46-40$ & $\mathrm{Mg}-\mathrm{Hb}$ & \\
\hline 55 & $52 \mathrm{R}-6,51-53$ & 8 & & 60 & tuff & & & & & $64 / 2$ & & & \\
\hline 56 & $72 \mathrm{R}-1,30-32$ & 8 & $20-70-10$ & 40 & tuff & sh & $30-60$ & (70) & & & & & alt. glass \\
\hline \multirow[t]{2}{*}{57} & $72 \mathrm{R}-1,55-58$ & 8 & $20-70-10$ & 30 & tuff & sh, gr & $30-70$ & (70) & $7-2 / 63-65$ & & & & alt. glass \\
\hline & $769 \mathrm{~A}$ & & & & & & & & & & & & \\
\hline 58 & $3 \mathrm{H}-4,102-103$ & 2 & $70-30-0$ & 70 & H & sc, bw & $100-160$ & $54.19-56.65$ & & & & & \\
\hline 59 & $4 \mathrm{H}-4,146-147$ & 2 & $70-30-3$ & 80 & $\mathrm{H}$ & sc, bw & $60-250$ & $53.82-56.26$ & & $64-55 / 1.3-2.5$ & & & \\
\hline \multirow[t]{2}{*}{60} & $5 \mathrm{H}-5,60-62$ & 2 & $50-50-0$ & 30 & D & sh & $50-180$ & $61.59-66.44$ & & $58-18 / 1.1-17.7$ & & $\mathrm{Mg}-\mathrm{Hb}$ & Mt (3.2-4.3) \\
\hline & $769 \mathrm{~B}$ & & & & & & & & & & & & \\
\hline 61 & $2 \mathrm{H}-7,28-30$ & 1 & $60-40-0$ & 40 & D & sh & $30-100(200)$ & $76.47-78.36$ & & $35-28 / 1.9-2.3$ & & & \\
\hline 62 & $3 \mathrm{H}-6,25-27$ & 2 & $90-10-0$ & 90 & $\mathrm{~T}$ & bw, mp, sh & $100-250$ & $56.08-66.34$ & & $67-26 / 1.6-7.2$ & $77 / 1+43 / 39$ & & Mt (7.8) \\
\hline 63 & $4 \mathrm{H}-1,53-55$ & 2 & $70-30-0$ & 70 & $\mathrm{~T}$ & bw, sc, sh & $50-250$ & $55.67-58.20$ & & $83 / 0.4$ & & & \\
\hline 64 & $5 \mathrm{H}-4,82-84$ & 2 & $40-40-20$ & 30 & D & sh, bw & $25-150$ & $55.47-74.00$ & & $71 / 0.7$ & $44 / 42$ & $\mathrm{Mg}-\mathrm{Hb}$ & \\
\hline 65 & $9 \mathrm{H}-7,29-31$ & 3 & $95-5-0$ & 95 & $\mathrm{H}$ & bw, sh & $100-400$ & 74.73-75.71 & & $52 / 0.9$ & $74-73 / 2.1-7.0$ & & \\
\hline 66 & $10 \mathrm{H}-5,102-104$ & 3 & $80-20-0$ & 70 & $\mathrm{~T}$ & sh, sc & $50-180$ & $59.83-65.30$ & & $41 / 2.6$ & & & \\
\hline 67 & $12 \mathrm{H}-2,4-6$ & $3-4$ & $90-10-0$ & 70 & $\mathrm{~T}$ & $\mathrm{sh}, \mathrm{mp}, \mathrm{sc}$ & $100-300$ & $56.14-67.28$ & 0/98 & $30 / 5.1$ & & & \\
\hline 68 & $30 X-2,125-127$ & 7 & $20-50-30$ & $\mathrm{n}$ & & & & & $1.1 / 58-60$ & $45 / 0.4$ & & $\mathrm{Mg}-\mathrm{Hb}$ & Mt (8.2) \\
\hline \multirow[t]{2}{*}{69} & $30 \mathrm{X}-\mathrm{cc}, 37-39$ & 7 & $50-40-10$ & $\mathrm{n}$ & & & & & & $62 / 1.0$ & & & Mt (7.3) \\
\hline & $769 \mathrm{C}$ & & & & & & & & & & & & \\
\hline 70 & $2 \mathrm{R}-6,7-9$ & 7 & $80-20-0$ & 40 & D & $\mathrm{sh}, \mathrm{mp}$ & $30-200(300)$ & (65) & 0/98 & & $43 / 42$ & $\mathrm{Mg}-\mathrm{Hb}$ & smect \\
\hline
\end{tabular}

Abbreviations: tr, trace; $\mathrm{n}$, none; sh, shards, bw, bubble-wall fragments; mp, micropumices; gr, grains; sc, microscoria. Size of glass in $\mu \mathrm{m}$. Mg-Hb, Mg-hornblende; $\mathrm{P}-\mathrm{Hb}$, pargasitic hornblende; Ts-Mg-Hb, tschermakitic or $\mathrm{Mg}$-hornblende; $\mathrm{Ab}$, albite; alt, altered; $\mathrm{Bi}$, biotite $\left(\mathrm{Mg} / \mathrm{Mg}+\mathrm{Fe}^{2+}+\mathrm{Mn} \%\right.$, $\left.\mathrm{TiO}_{2} \%\right) ; \mathrm{Ep}$, epidote; Ilm, ilmenite $\left(\mathrm{TiO}_{2} \%\right)$; $\mathrm{Mt}^{2}$, magnetite ( $\left.\mathrm{TiO} \mathrm{O}_{2} \%\right) ; \mathrm{Ol}$, olivine $(\mathrm{Forsterite} \%)$; $\mathrm{Qtz}$, quartz; smect, smectite. 
Table 2. Chemical analyses and norms of glasses. Total $\mathrm{Fe}$ as $\mathrm{Fe}_{2} \mathrm{O}_{3}$ : calculated $\mathrm{FeO} / \mathrm{FeO}+\mathrm{Fe}_{2} \mathrm{O}_{3}$ $=0.85$ for norm calculation.

\begin{tabular}{|c|c|c|c|c|c|c|c|c|c|c|}
\hline & 1 & $\begin{array}{c}\mathrm{A} \\
2\end{array}$ & 3 & 4 & 5 & 6.1 & 6.2 & 7 & 8 & 9 \\
\hline $\mathrm{SiO}_{2}$ & 77.41 & 77.91 & 78.55 & 74.50 & 74.56 & 59.12 & 67.00 & 76.11 & 75.54 & 77.70 \\
\hline $\mathrm{TiO}_{2}^{2}$ & 0.13 & 0.07 & 0.09 & 0.36 & 0.32 & 0.86 & 0.40 & 0.24 & 0.18 & 0.07 \\
\hline $\mathrm{Al}_{2} \mathrm{O}_{3}$ & 12.94 & 12.88 & 12.55 & 14.03 & 13.82 & 17.61 & 16.92 & 13.45 & 13.72 & 13.95 \\
\hline $\mathrm{Fe}_{2} \mathrm{O}_{3}$ & 1.03 & 0.88 & 0.94 & 1.75 & 1.72 & 6.65 & 3.43 & 1.63 & 1.38 & 0.95 \\
\hline $\mathrm{FeO}$ & nd & nd & nd & nd & nd & nd & nd & nd & nd & nd \\
\hline $\mathrm{MnO}$ & 0.07 & 0.10 & 0.13 & 0.05 & 0.15 & 0.18 & 0.20 & 0.10 & 0.17 & 0.08 \\
\hline $\mathrm{MgO}$ & 0.22 & 0.25 & 0.22 & 0.39 & 0.39 & 2.03 & 0.50 & 0.30 & 0.25 & 0.27 \\
\hline $\mathrm{CaO}$ & 1.12 & 1.11 & 0.98 & 1.36 & 1.31 & 4.81 & 1.23 & 1.47 & 0.98 & 1.34 \\
\hline $\mathrm{Na}_{2} \mathrm{O}$ & 3.96 & 3.80 & 3.24 & 4.12 & 4.26 & 4.87 & 5.07 & 3.80 & 3.94 & 3.60 \\
\hline $\mathrm{K}_{2} \mathrm{O}$ & 3.12 & 3.00 & 3.30 & 3.43 & 3.46 & 3.87 & 5.25 & 2.90 & 3.83 & 2.04 \\
\hline TÖTAL & 100.00 & 100.00 & 100.00 & 100.00 & 100.00 & 100.00 & 100.00 & 100.00 & 100.00 & 100.00 \\
\hline IL & 0.25 & 0.13 & 0.18 & 0.69 & 0.61 & 1.72 & 0.78 & 0.46 & 0.34 & 0.13 \\
\hline MT & 0.22 & 0.19 & 0.20 & 0.38 & 0.38 & 1.53 & 0.77 & 0.36 & 0.30 & 0.20 \\
\hline OR & 18.43 & 17.66 & 19.34 & 20.37 & 20.65 & 24.07 & 31.84 & 17.1 & 22. & 11.77 \\
\hline $\mathrm{AB}$ & 33.50 & 32.03 & 27.17 & 35.06 & 36.42 & 43.38 & 44.05 & 32.20 & 33.33 & 29.76 \\
\hline AN & 5.56 & 5.49 & 4.82 & 6.79 & 5.44 & 15.54 & 6.25 & 7.30 & 4.88 & 6.49 \\
\hline DI & nd & nd & nd & nd & nd & 7.99 & nd & nd & nd & nd \\
\hline HY & 0.83 & 0.86 & 0.85 & 1.30 & 1.40 & 4.49 & 2.44 & 1.21 & 1.09 & 0.90 \\
\hline C & 2.03 & 2.72 & 3.71 & 2.13 & 1.39 & nd & 1.36 & 2.78 & 2.64 & 6.62 \\
\hline Q & 39.19 & 40.92 & 43.74 & 33.28 & 32.60 & 1.29 & 12.51 & 38.55 & 34.76 & 44.13 \\
\hline \multirow[t]{2}{*}{ TL } & 100.00 & 100.00 & 100.00 & 100.00 & 100.00 & 100.00 & 100.00 & 100.00 & 100.00 & 100.00 \\
\hline & 10 & 11 & 12 & 13 & 14.1 & 14.2 & 14.3 & 15 & 25 & 28 \\
\hline $\mathrm{SiO}_{2}$ & 77.73 & 78.53 & 76.58 & 61.39 & 63.24 & 66.25 & 70.4 & 77.39 & 77.5 & 76.21 \\
\hline $\mathrm{TiO}_{2}$ & 0.09 & 0.12 & 0.15 & 0.68 & 0.85 & 0.64 & 0.88 & 0.09 & 0.05 & 0.01 \\
\hline $\mathrm{Al}_{2} \mathrm{O}_{3}$ & 13.61 & 12.34 & 13.52 & 16.08 & 14.72 & 15.51 & 14.00 & 13.09 & 13.39 & 14.34 \\
\hline $\mathrm{Fe}_{2} \mathrm{O}_{3}$ & 1.02 & 0.86 & 1.08 & 6.32 & 9.02 & 5.51 & 4.78 & 1.05 & 0.40 & 0.16 \\
\hline $\mathrm{FeO}$ & nd & nd & nd & nd & nd & nd & nd & nd & nd & nd \\
\hline $\mathrm{MnO}$ & 0.13 & 0.13 & 0.07 & 0.16 & 0.35 & 0.32 & 0.04 & 0.08 & 0.10 & 0.09 \\
\hline $\mathrm{gO}$ & 0.22 & 0.13 & 0.15 & 2.30 & 1.5 & 1.0 & 0.2 & 0.18 & 0.03 & 0.18 \\
\hline $\mathrm{CaO}$ & 1.21 & 0.84 & 1.00 & 3.66 & 3.97 & 2.6 & 1.70 & 1.0 & 1.93 & 2.27 \\
\hline $\mathrm{Na}_{2} \mathrm{O}$ & 3.49 & 3.31 & 3.25 & 4.48 & 3.66 & 4.23 & 3.67 & 3.15 & 4.02 & 3.73 \\
\hline $\mathrm{K}_{2} \mathrm{O}$ & 2.50 & 3.74 & 4.20 & 4.92 & 2.63 & 3.79 & 4.24 & 3.97 & 2.49 & 3.02 \\
\hline TÓTAL & 100.00 & 100.00 & 100.00 & 100.00 & 100.00 & 100.00 & 100.00 & 100.00 & 100.00 & 100.00 \\
\hline IL & 0.17 & 0.23 & 0.28 & 1.37 & 1.77 & 1.29 & 1.73 & 0.17 & 0.10 & 0.02 \\
\hline MT & & & & & & 7 & & & & 0.03 \\
\hline OR & 14.50 & 21.99 & 24.60 & 30.66 & 16.91 & 23.59 & 25.89 & 23.2 & 14.67 & 17.74 \\
\hline $\mathrm{AB}$ & 28.99 & 27.87 & 27.27 & 39.94 & 33.69 & 37.72 & 32.10 & 26.45 & 34.00 & 31.40 \\
\hline AN & 5.89 & 4.15 & 4.92 & 9.7 & 17.3 & 12.8 & 8.72 & 4.92 & 9.57 & 11.21 \\
\hline DI & nd & nd & nd & 7.90 & 3. & 1.03 & nd & nd & nd & nd \\
\hline HY & 0.87 & 0.64 & 0.72 & 4.89 & 6.41 & 4.23 & 2.32 & 0.80 & 0.28 & 0.43 \\
\hline C & 5.82 & 2.63 & 3.59 & nd & nd & nd & 0.58 & 3.56 & 1.13 & 1.61 \\
\hline Q & 43.54 & 42.30 & 38.38 & 4.06 & 18.43 & 18.03 & 27.57 & 40.59 & 40.16 & 37.55 \\
\hline TL & 100.00 & 100.00 & 100.00 & 100.00 & 100.00 & 100.00 & 100.00 & 100.00 & 100.00 & 100.00 \\
\hline
\end{tabular}

\begin{tabular}{|c|c|c|c|c|c|c|c|c|c|c|}
\hline & 30 & $\begin{array}{c}\text { B } \\
31\end{array}$ & 32 & 33 & 34 & 35.1 & 35.2 & 35.3 & 36 & 38 \\
\hline $\mathrm{SiO}_{2}$ & 77.57 & 77.72 & 78.36 & 76.69 & 58.74 & 57.40 & 63.29 & 66.92 & 55.92 & 77.76 \\
\hline $\mathrm{TiO}_{2}$ & 0.10 & 0.06 & 0.19 & 0.22 & 0.84 & 0.89 & 0.66 & 0.37 & 0.96 & 0.24 \\
\hline $\mathrm{Al}_{2} \mathrm{O}_{3}$ & 13.08 & 12.90 & 12.15 & 12.98 & 17.44 & 17.15 & 17.43 & 17.14 & 16.41 & 12.18 \\
\hline $\mathrm{Fe}_{2} \mathrm{O}_{3}$ & 0.74 & 0.22 & 1.28 & 1.02 & 6.75 & 8.77 & 4.08 & 2.59 & 9.35 & 1.66 \\
\hline $\mathrm{FeO}$ & nd & nd & nd & nd & nd & nd & nd & nd & nd & nd \\
\hline $\mathrm{MnO}$ & 0.11 & 0.19 & 0.07 & 0.07 & 0.15 & 0.11 & 0.15 & 0.13 & 0.19 & 0.12 \\
\hline $\mathrm{MgO}$ & 0.18 & 0.05 & 0.20 & 0.23 & 2.32 & 2.52 & 1.19 & 0.39 & 3.26 & 0.23 \\
\hline $\mathrm{CaO}$ & 0.92 & 1.02 & 1.24 & 1.12 & 4.91 & 5.73 & 2.78 & 1.10 & 6.36 & 1.34 \\
\hline $\mathrm{Na}_{2} \mathrm{O}$ & 3.87 & 3.93 & 3.61 & 3.89 & 5.12 & 4.05 & 5.59 & 5.48 & 4.21 & 3.80 \\
\hline $\mathrm{K}_{2} \mathrm{O}$ & 3.43 & 3.89 & 2.90 & 3.78 & 3.71 & 3.38 & 4.83 & 5.88 & 3.34 & 2.67 \\
\hline TOTAL & 100.00 & 100.00 & 100.00 & 100.00 & 100.00 & 100.00 & 100.00 & 100.00 & 100.00 & 100.00 \\
\hline IL & 0.19 & 0.12 & 0.35 & 0.42 & 1.67 & 1.82 & 1.29 & 0.72 & 1.90 & 0.46 \\
\hline MT & & & & & & & 0.92 & 0.58 & 2.13 & 0.37 \\
\hline OR & 20.15 & 22.97 & 17.20 & 22.43 & 22.90 & 21.48 & 29.40 & 35.54 & 20.61 & 15.92 \\
\hline $\mathrm{AB}$ & 32.57 & 33.29 & 30.67 & 33.10 & 45.28 & 36.87 & 48.73 & 47.44 & 37.24 & 32.45 \\
\hline AN & 4.54 & 5.08 & 6.19 & 5.57 & 14.24 & 20.05 & 8.45 & 4.91 & 16.70 & 6.71 \\
\hline DI & nd & nd & nd & nd & 9.38 & 8.76 & 4.80 & 0.55 & 13.61 & nd \\
\hline HY & 0.67 & 0.29 & .88 & 0.78 & 3.89 & 6.34 & 2.54 & 1.67 & 3.64 & 1.13 \\
\hline $\mathrm{OL}$ & nd & nd & nd & nd & 1.10 & nd & nd & nd & 4.17 & nd \\
\hline C & 2.64 & 0.73 & 1.62 & 0.91 & nd & nd & nd & nd & nd & 1.22 \\
\hline Q & 39.08 & 37.48 & 42.81 & 36.57 & nd & 2.63 & 3.86 & 8.60 & nd & 41.76 \\
\hline \multirow[t]{2}{*}{ TL } & 100.00 & 100.00 & 100.00 & 100.00 & 100.00 & 100.00 & 100.00 & 100.00 & 100.00 & 100.00 \\
\hline & 39 & 41 & 43 & 45 & 47 & 49 & 50 & 51 & 52 & 53 \\
\hline $\mathrm{SiO}_{2}$ & & & & & 78.22 & & 78.23 & 77.42 & 67.95 & \\
\hline $\mathrm{TiO}_{2}$ & 0.25 & 0.10 & 0.13 & 0.20 & 0.17 & 0.24 & 0.10 & 0.13 & 0.11 & 0.08 \\
\hline
\end{tabular}


Table 2 (continued).

\begin{tabular}{lcccccccccc}
\hline $\mathrm{Al}_{2} \mathrm{O}_{2}$ & 13.74 & 11.39 & 14.01 & 14.40 & 14.18 & 14.11 & 14.23 & 13.97 & 18.79 & 16.19 \\
$\mathrm{Fe}_{2} \mathrm{O}_{3}$ & 1.83 & 0.32 & 1.40 & 1.33 & 1.56 & 1.53 & 1.38 & 1.41 & 0.53 & 0.13 \\
$\mathrm{FeO}$ & nd & nd & nd & \multicolumn{1}{c}{ nd } & nd & nd & nd & nd & \multicolumn{1}{c}{ nd } & nd \\
$\mathrm{MnO}$ & 0.13 & 0.13 & 0.06 & 0.10 & 0.05 & 0.22 & 0.06 & 0.12 & 0.05 & 0.06 \\
$\mathrm{MgO}$ & 0.48 & 0.19 & 0.15 & 0.13 & 0.17 & 0.10 & 0.11 & 0.14 & 0.29 & 0.28 \\
$\mathrm{CaO}$ & 1.50 & 0.72 & 0.97 & 0.98 & 1.04 & 0.85 & 0.95 & 1.00 & 3.82 & 2.16 \\
$\mathrm{Na}_{2} \mathrm{O}$ & 4.67 & 3.62 & 2.68 & 2.78 & 2.39 & 2.73 & 2.78 & 3.38 & 3.53 & 3.30 \\
$\mathrm{~K}_{2} \mathrm{O}$ & 3.37 & 4.29 & 2.31 & 2.28 & 2.22 & 2.61 & 2.16 & 2.43 & 4.93 & 3.74 \\
$\mathrm{TOTAL}$ & 100.00 & 100.00 & 100.00 & 100.00 & 100.00 & 100.00 & 100.00 & 100.00 & 100.00 & 100.00 \\
$\mathrm{IL}$ & 0.49 & 0.19 & 0.24 & 0.36 & 0.31 & 0.44 & .18 & 0.24 & 0.21 & .15 \\
$\mathrm{MT}$ & 0.41 & .07 & 0.29 & 0.28 & 0.33 & 0.32 & 0.29 & 0.30 & 0.12 & 0.03 \\
$\mathrm{OR}$ & 20.28 & 25.36 & 13.11 & 12.90 & 12.54 & 14.84 & 12.22 & 13.98 & 29.11 & 21.56 \\
$\mathrm{AB}$ & 40.22 & 30.65 & 21.78 & 22.52 & 19.33 & 22.24 & 22.54 & 27.86 & 29.85 & 27.25 \\
$\mathrm{AN}$ & 6.66 & 2.19 & 4.62 & 4.65 & 4.93 & 4.06 & 4.52 & 4.83 & 18.94 & 10.46 \\
$\mathrm{DI}$ & 0.76 & 1.17 & nd & nd & nd & nd & nd & nd & nd & nd \\
$\mathrm{HY}$ & 1.44 & 0.20 & 0.85 & 0.77 & 0.92 & 0.88 & 0.80 & 0.89 & 0.70 & 0.50 \\
$\mathrm{OL}$ & nd & nd & nd & nd & nd & nd & nd & nd & nd & nd \\
$\mathrm{C}$ & nd & nd & 10.27 & 10.69 & 11.40 & 10.12 & 10.73 & 7.73 & 1.40 & 5.44 \\
$\mathrm{Q}$ & 29.74 & 40.17 & 48.84 & 47.83 & 50.25 & 47.10 & 48.73 & 44.16 & 19.67 & 34.62 \\
$\mathrm{TL}$ & 100.00 & 100.00 & 100.00 & 100.00 & 100.00 & 100.00 & 100.00 & 100.00 & 100.00 & 100.00
\end{tabular}

\begin{tabular}{|c|c|c|c|c|c|c|c|c|c|c|}
\hline & 61 & $\begin{array}{c}C \\
62.1\end{array}$ & 62.2 & 58 & 63 & 59 & 64.1 & 64.2 & 64.3 & 60 \\
\hline $\mathrm{SiO}_{2}$ & 77.94 & 56.46 & 66.26 & 55.05 & 57.32 & 55.00 & 55.47 & 64.13 & 73.99 & 66.44 \\
\hline $\mathrm{TiO}_{2}^{2}$ & 0.08 & 1.07 & 0.34 & 0.99 & 0.82 & 0.99 & 1.18 & 1.00 & 0.14 & 0.96 \\
\hline $\mathrm{Al}_{2} \mathrm{O}_{3}$ & 12.58 & 16.52 & 17.78 & 16.95 & 16.41 & 17.83 & 14.17 & 14.65 & 14.38 & 16.31 \\
\hline $\mathrm{Fe}_{2} \mathrm{O}_{3}$ & 0.83 & 8.67 & 2.55 & 9.23 & 7.73 & 7.64 & 11.22 & 6.16 & 1.36 & 3.29 \\
\hline $\mathrm{FeO}$ & nd & nd & nd & nd & nd & nd & nd & nd & nd & nd \\
\hline $\mathrm{MnO}$ & 0.09 & 0.10 & 0.02 & 0.17 & 0.15 & 0.11 & 0.19 & 0.12 & 0.09 & 0.23 \\
\hline $\mathrm{MgO}$ & 0.15 & 2.92 & 0.36 & 3.13 & 2.63 & 3.19 & 4.68 & 1.63 & 0.21 & 1.49 \\
\hline $\mathrm{CaO}$ & 1.12 & 5.89 & 1.15 & 7.16 & 5.84 & 6.18 & 6.65 & 4.36 & 1.20 & 2.28 \\
\hline $\mathrm{Na}_{2} \mathrm{O}$ & 3.57 & 5.27 & 5.65 & 4.26 & 4.97 & 5.20 & 4.35 & 4.86 & 4.13 & 5.67 \\
\hline $\mathrm{K}_{2} \mathrm{O}$ & 3.65 & 3.10 & 5.89 & 3.07 & 4.13 & 3.87 & 2.07 & 3.09 & 4.50 & 3.33 \\
\hline TOัTAL & 100.00 & 100.00 & 100.00 & 100.00 & 100.00 & 100.00 & 100.00 & 100.00 & 100.00 & 100.00 \\
\hline IL & 0.14 & 2.05 & 0.66 & 1.91 & 1.57 & 1.89 & 2.44 & 1.97 & 0.27 & 1.88 \\
\hline MT & 0.18 & 1.90 & 0.57 & 2.04 & 1.70 & 1.67 & 2.65 & 1.39 & 0.30 & 0.74 \\
\hline OR & 21.61 & 18.44 & 35.58 & 18.40 & 24.56 & 22.88 & 13.25 & 18.88 & 26.79 & 20.30 \\
\hline $\mathrm{AB}$ & 30.23 & 42.28 & 48.88 & 36.58 & 38.25 & 31.49 & 39.87 & 42.52 & 35.22 & 49.44 \\
\hline AN & 5.57 & 12.35 & 5.83 & 18.33 & 10.34 & 13.92 & 14.09 & 9.34 & 6.00 & 9.49 \\
\hline DI & nd & 14.28 & nd & 14.81 & 15.74 & 14.12 & 18.21 & 10.83 & nd & 1.85 \\
\hline HY & 0.66 & nd & 1.67 & 0.69 & nd & nd & 7.90 & 2.61 & 0.99 & 3.24 \\
\hline $\mathrm{OL}$ & nd & 7.28 & nd & 7.24 & 5.63 & 7.25 & 1.60 & nd & nd & nd \\
\hline NE & nd & 1.42 & nd & nd & 2.21 & 6.79 & nd & nd & nd & nd \\
\hline C & 1.44 & nd & 0.04 & nd & nd & nd & nd & nd & 1.08 & nd \\
\hline Q & 40.17 & nd & 6.77 & nd & nd & nd & nd & 12.46 & 29.36 & 13.06 \\
\hline TL & 100.00 & 100.00 & 100.00 & 100.00 & 100.00 & 100.00 & 100.00 & 100.00 & 100.00 & 100.00 \\
\hline
\end{tabular}

\begin{tabular}{|c|c|c|c|c|c|}
\hline & 65 & 66.1 & 66.2 & 67.1 & 67.2 \\
\hline $\mathrm{SiO}_{2}$ & 75.27 & 59.94 & 64.33 & 56.14 & 66.25 \\
\hline $\mathrm{TiO}_{2}^{2}$ & 0.25 & 0.86 & 0.72 & 1.08 & 0.53 \\
\hline $\mathrm{Al}_{2} \mathrm{O}_{3}$ & 13.96 & 17.33 & 16.79 & 17.32 & 16.75 \\
\hline $\mathrm{Fe}_{2} \mathrm{O}_{3}$ & 1.37 & 7.00 & 4.99 & 9.10 & 4.60 \\
\hline $\mathrm{FeO}$ & nd & nd & nd & nd & nd \\
\hline $\mathrm{MnO}$ & 0.13 & 0.28 & 0.25 & 0.22 & 0.01 \\
\hline $\mathrm{MgO}$ & 0.26 & 2.06 & 1.49 & 3.05 & 0.67 \\
\hline $\mathrm{CaO}$ & 1.12 & 4.59 & 3.16 & 6.04 & 2.20 \\
\hline $\mathrm{Na}_{2} \mathrm{O}$ & 4.05 & 4.44 & 4.40 & 4.51 & 4.63 \\
\hline $\mathrm{K}_{2} \mathrm{O}$ & 3.59 & 3.50 & 3.86 & 2.54 & 4.38 \\
\hline TŌTAL & 100.00 & 100.00 & 100.00 & 100.00 & 100.00 \\
\hline IL & 0.48 & 1.74 & 1.44 & 2.23 & 1.04 \\
\hline MT & 0.30 & 1.63 & 1.15 & 2.14 & 1.04 \\
\hline OR & 21.19 & 22.03 & 24.00 & 16.24 & 26.83 \\
\hline AB & 34.24 & 40.03 & 39.19 & 41.25 & 40.64 \\
\hline $\mathrm{AN}$ & 5.55 & 18.14 & 15.44 & 21.11 & 11.34 \\
\hline DI & nd & 5.10 & 0.89 & 9.43 & nd \\
\hline HY & 1.03 & 5.59 & 4.61 & 7.27 & 3.12 \\
\hline OL & nd & nd & nd & nd & nd \\
\hline NE & nd & nd & nd & nd & nd \\
\hline C & 2.75 & nd & nd & nd & 0.84 \\
\hline Q & 34.47 & 5.74 & 13.28 & 0.32 & 15.16 \\
\hline TL & 100.00 & 100.00 & 100.00 & 100.00 & 100.00 \\
\hline
\end{tabular}

A. Sites 767, 770. B. Site 768. C. Site 769. Samples are in chronologic order. The table presents one averaged analysis for each homogeneous sample and two or three averaged or representative analyses for heterogeneous samples. 


\section{Amphiboles and Biotite}

Amphiboles are common minerals in many layers, as pale to dark brown-green fragments. They have been analyzed in 26 samples. All proved to be calcic amphiboles, classified using the Leake (1978) nomenclature (Table 1). Their low Al and Ti-contents are consistent with sub-alkaline characters (Fig. 7). Most of the amphiboles are $\mathrm{Mg}$-hornblendes associated with dacitic and rhyolitic glasses. Pargasitic hornblendes were determined in Site 767 Pliocene strata, in association with andesitic glasses (samples 13,14). A reddish and high- $\mathrm{Mg}$ hornblende has been found in Site 767 Pliocene-Pleistocene strata and associated with feldspar, orthopyroxene, and rare magnetite and epidote, indicating proximity of mafic volcanic terrane. Assuming that amphibole crystallized with all the phases in the lava plus melt, we use the empirical geobarometer of Hammarstrom and Zen (1986): Mg-hornblendes are related to low-pressure crystallization (averaged total Alcontent indicates $2.6 \pm 3 \mathrm{~kb}$ ), whereas pargasitic hornblendes correspond to higher pressure crystallization $(6.1 \pm 3 \mathrm{~kb})$. The Hollister et al, (1987) equation gives the same relative results, respectively $2.5 \pm 1.5 \mathrm{~kb}$ and $6.5 \pm 1.5 \mathrm{~kb}$.

Biotite is less common, as generally oxidized brown flakes. Fresh biotites and $\mathrm{Mg}$-biotites were analyzed in three sam-
Table 3. Minor-element chemical analysis of Sample 124-767B-3H-1,88-90. Inductively coupled plasma emission spectrometry. Elements in parts per million.

\begin{tabular}{llllll}
\hline $\mathrm{La}=$ & 12.80 & $\mathrm{Ba}$ & $=$ & 310 \\
$\mathrm{Ce}$ & 30.54 & $\mathrm{Be}$ & $=$ & 0.8 \\
$\mathrm{Nd}=$ & 13.86 & $\mathrm{Co}$ & $=$ & 180 \\
$\mathrm{Sm}=$ & 4.30 & $\mathrm{Cr}$ & $=$ & 12 \\
$\mathrm{Eu}=$ & 0.76 & $\mathrm{Cu}=$ & 54 \\
$\mathrm{Gd}=$ & 3.87 & $\mathrm{Ga}$ & $=$ & 9 \\
$\mathrm{Dy}=$ & 4.39 & $\mathrm{Ni}$ & $=$ & 26 \\
$\mathrm{Er}=$ & 2.76 & $\mathrm{Rb}$ & $=$ & 54 \\
$\mathrm{Yb}=$ & 3.08 & $\mathrm{Sc}$ & $=$ & 13.3 \\
$\mathrm{Lu}=$ & 0.62 & $\mathrm{Sr}$ & $=$ & 149 \\
& & $\mathrm{~V}$ & $=$ & 16 \\
& & $\mathrm{Y}$ & $=$ & 35.85 \\
& & $\mathrm{Zn}$ & $=57$ \\
& & $\mathrm{Zr}$ & $=$ & 183 \\
\hline
\end{tabular}

ples. The $100 \mathrm{Mg} / \mathrm{Mg}+\mathrm{Fe}^{2+}$ ratio ranges from $58 \%$ to $66 \%$ in "rhyolitic" beds, and to $71 \%$ in "mafic" beds, indicating higher $\mathrm{PH}_{2} \mathrm{O}$ and $\mathrm{fO}_{2}$ conditions (Wones and Eugster, 1965). $\mathrm{TiO}_{2}$ contents vary from $3.2 \%$ to $3.9 \%$.
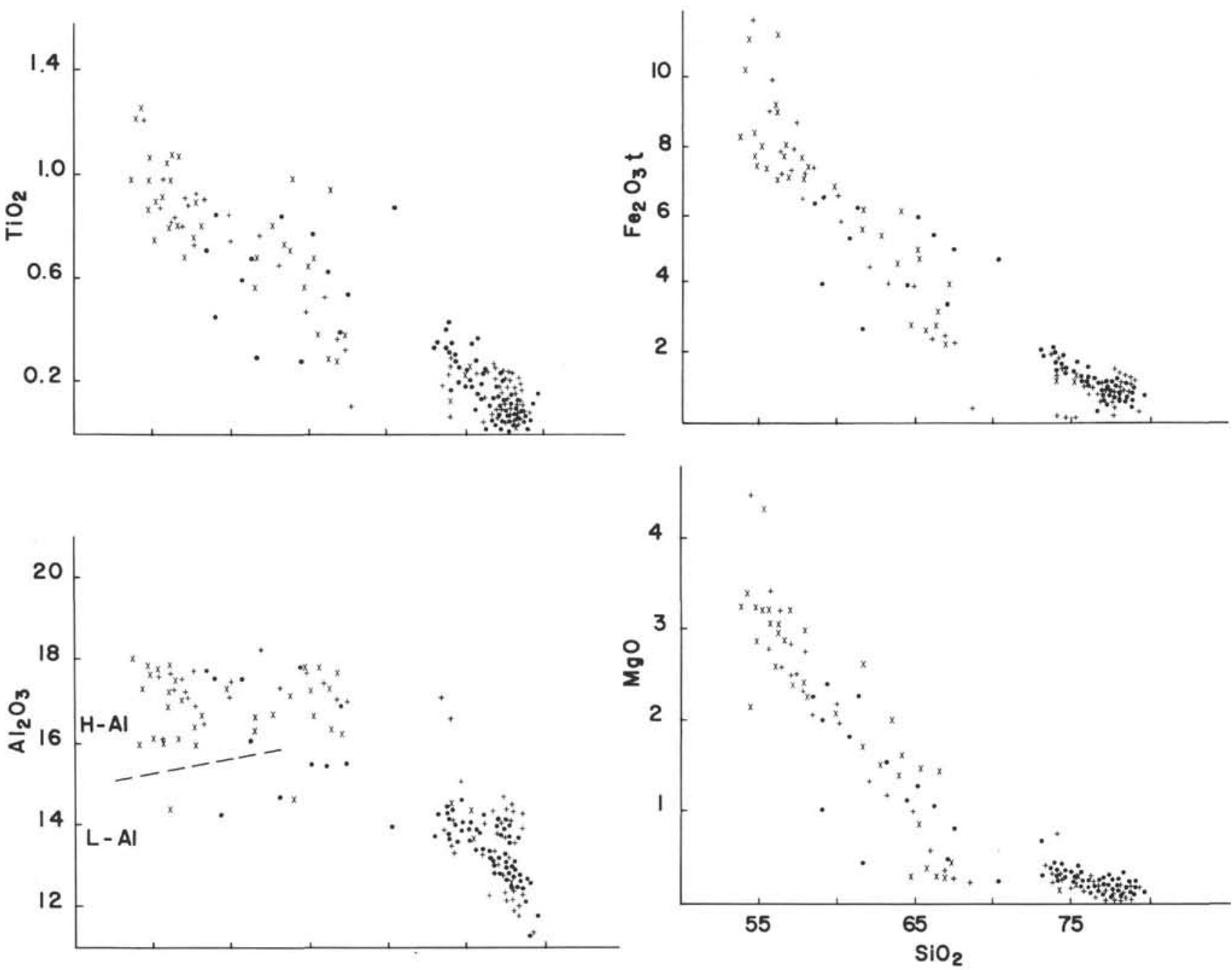

Figure 2. $\mathrm{SiO}_{2}$ vs. oxides variation diagrams. Solid circles: Sites 767 and 770; cross: Site 768; X: Site 769; L-Al and H-Al, low- and highalumina compositions. L-K, M-K, and H-K, Low-, medium-, and high-potassic compositions. B-And, basaltic andesite; And, andesite; $\mathrm{DC}$, dacite; RH, rhyolite. 


\section{Oxides}

Magnetite occurs as epiclastic and oxidized grains, or as tiny fragments and euhedral pyroclastic crystals that were analyzed in thirteen samples. $\mathrm{TiO}_{2}$-contents vary from $3.2 \%$ to $9.5 \%$ in "rhyolitic" beds, and to $15.2 \%$ in "andesitic" beds.

Ilmenite consists of rare fragments that were analyzed in only four samples. They have high $\mathrm{TiO}_{2}$ content $(42 \%-47 \%)$ in "acidic" beds and low $\mathrm{TiO}_{2}$ content $(26 \%-27 \%)$ in "mafic" beds, when coexisting with Ti-rich magnetite and biotite.

Two coexisting iron-titanium oxides were found in two samples. We calculated thermometry and oxygen barometry, using phase recalculations of Stormer (1983) and formulations of Andersen and Lindsley (1988), with the following results:

1. Sample $124-768 \mathrm{~B}-16 \mathrm{H}-2,49-51 \mathrm{~cm}: \mathrm{T}=842^{\circ} \mathrm{C}$, $\log (10)$ $\mathrm{fO}_{2}=-10.6$. These data are consistent with dacitic liquid composition.

2. Sample $124-767 \mathrm{C}-6 \mathrm{R}-1,17-19 \mathrm{~cm}: \mathrm{T}=1035^{\circ} \mathrm{C}, \log (10)$ $\mathrm{fO}_{2}=-8.4$. The high temperature and very high oxygen fugacity may be related to volatile-rich andesitic magma.

\section{Miscellaneous Minerals}

Detrital epiclastic olivine Fo 79.5 was found in Sample 5 (124-767B-1H-3, 77-79 cm) together with CPX, Ca-plagioclase, and $\mathrm{Mg}$-hornblende, indicating andesitic paragenesis. But coexisting glass shards are rhyolitic in composition. Andesitic scoria are known in slightly older layers at the same site (124-767B-2H-7, 36-38 cm). Thus, a proximate andesitic source does exist. Detrital epidote pistacite $\left(12.67 \% \mathrm{Fe}_{2} \mathrm{O}_{3}\right)$ was analyzed in Sample 9 (124-767B-8H-4, 21-23 cm), a turbidite rhyolitic ash layer that indicates the proximity of metamorphic terrane.

\section{Petrologic Interpretation and Magmatic Affinities}

Chemical variations using all of the glass compositions are depicted in Figure 2. Because several samples contain differentiated basic to acidic glasses together with their minerals, an attempt was made to determine the petrological features that may express magmatic affinities. Distinctive chemical compositions are evidenced with alumina and alkalies vs. silica variations, whereas lime, magnesia, and iron exhibit common decreasing contents from basic to acidic compositions. Selected and averaged samples from Figure 2 are plotted on Figure 8: diagrams of $\mathrm{Al}_{2} \mathrm{O}_{3}, \mathrm{CaO}, \mathrm{Na}_{2} \mathrm{O}$ and $\mathrm{K}_{2} \mathrm{O}$ vs. $\mathrm{SiO}_{2}$. Tie-lines join glass composition types coexisting in ash beds, showing parallel or divergent trends. Dashed lines correspond to probable trends for acidic glasses. Two successive gross variations are distinguishable from andesitic to dacitic glasses, and from dacitic to rhyolitic glasses, with a drastic change at $\mathrm{SiO}_{2} 67 \%-68 \%$. Variations may be due (1) to fractional crystallization, (2) to magma mixing or mingling, (3) to crustal contamination, or (4) to any other secondary process (alkali loss in altered acidic glasses). Either crystal fractionation or crustal contamination may explain the potassium enrichment of some glasses of intermediate silica composition; it is impossible to decipher from the major
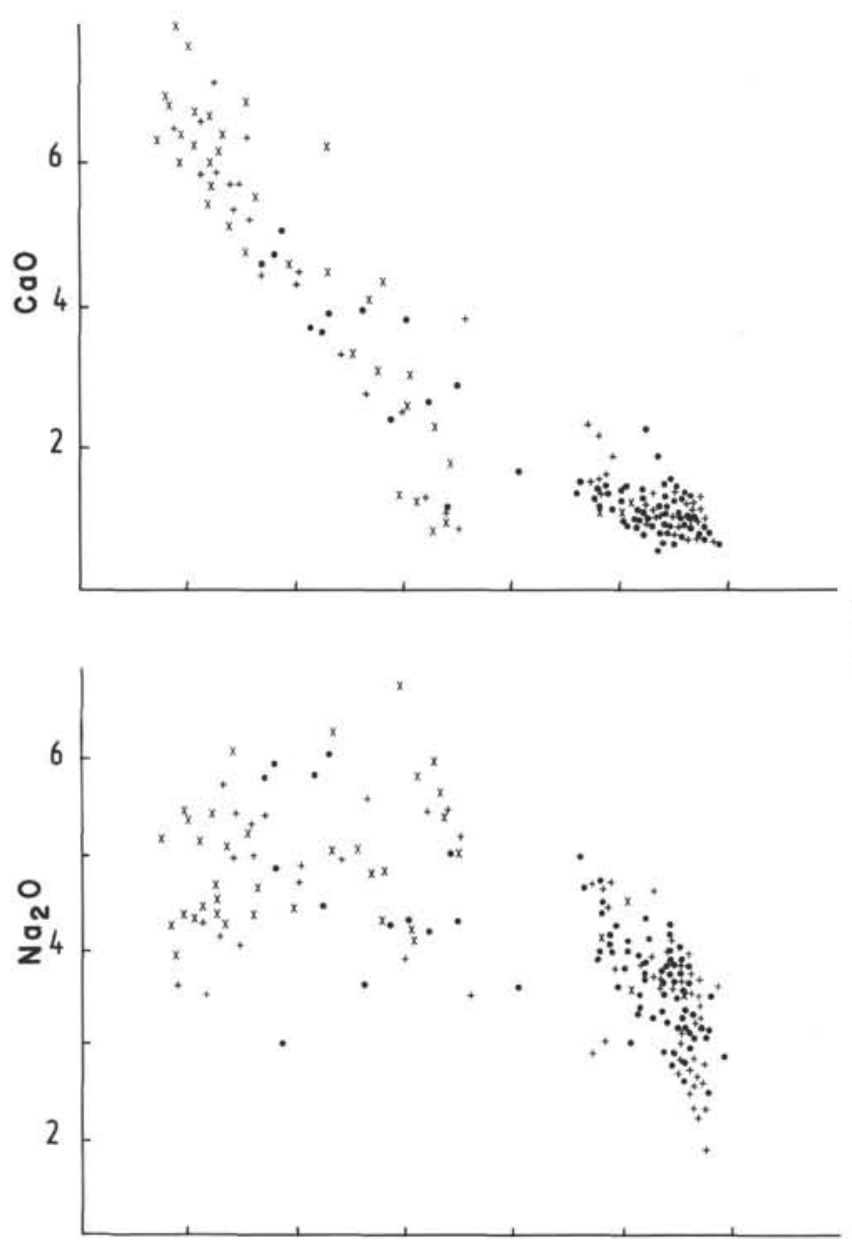

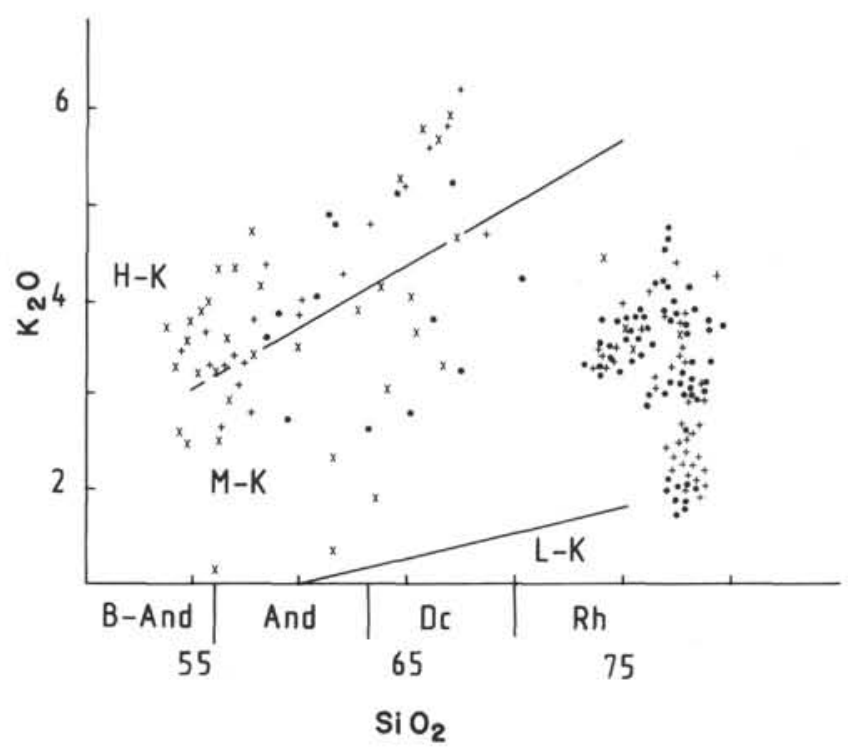

Figure 2 (continued). 


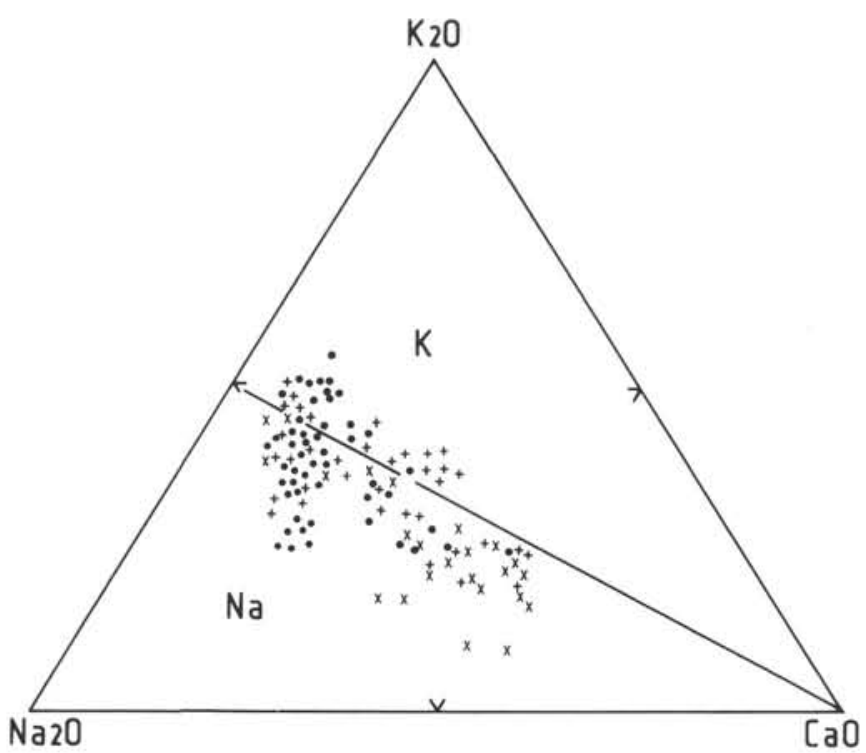

Figure 3. $\mathrm{CaO}-\mathrm{Na}_{2} \mathrm{O}-\mathrm{K}_{2} \mathrm{O}$ diagram; sodic $(\mathrm{Na})$ and potassic $(\mathrm{K})$ fields. Symbols as for Figure 2.

element data. Magmamixing and/or mingling is obvious, considering the very high range of feldspar compositions and the multicompositional ashes; however, in common calc-alkaline volcanoes, this affects the more-or-less differentiated magmas of a cogenetic suites.

By using coexisting mineral analyses and glass compositions, least-square-mass balance calculations can test chemical evolutions in term of fractionation of distinct magmatic series (Wright and Doherty, 1970); Table 5. Samples were selected to represent the different chemical variations in the age groups. When possible, the parent and daughter liquids are coexisting glasses from mixed ash layers having consistent compositional trends. At least they belong to eruptions close in age. Apparently successful calculations (low residuals) are a consequence of the least-squares approach by choosing appropriate phases. We assume that coexisting glasses and minerals in a discrete ash layer originated from a single volcanic system, but we do not know if the glasses are formally parent and daughter liquids. The Table 5 presents a summary of the result (completely documented calculations would give a fallacious impression of accuracy). Trends are numbered according to their increasing slope on an alumina/ silica diagram (Fig. 8). As a practical result, we distinguish two fractionation steps and three differentiation trends. This distinction brings an additional guide in ascribing ash deposits to volcanic belts.

\section{The First Fractionation Step}

This step, from andesites to dacites, involves clinopyroxenes (Wo 41, En 40-44), titanomagnetites, amphiboles (pargasitic hornblende and $\mathrm{Mg}$-hornblende), $\mathrm{Mg}$-biotites, and plagioclases (An 60-35). These results are only indicative of general evolution. Various calculations can be done, but not with very different solutions. We may distinguish three types of fractionation trend.

\section{Type A}

This is a major fractionation of amphibole, subordinate plagioclase of the low-temperature trend, and clinopyroxene. This is related to low-pressure crystallization of volatile-rich

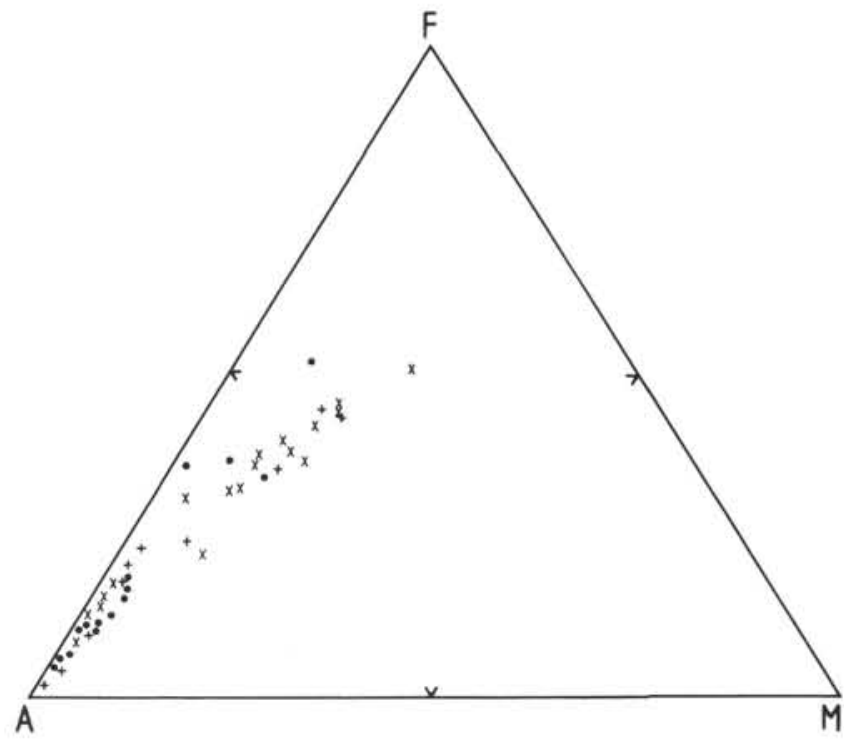

Figure 4. A-F-M diagram $\left(\mathrm{Na}_{2} \mathrm{O}+\mathrm{K}_{2} \mathrm{O}\right.$-total $\left.\mathrm{Fe}_{2} \mathrm{O}_{3}-\mathrm{MgO}\right)$. Averaged analytical data. Symbols as in figure 2 .

andesitic magma. These features, and low Al- and alkaliescontents (see above) characterize moderate potassic andesitic series of an island arc. The type-A magmatic products locate Site 767 in early Pliocene to early Pleistocene strata, and Site 769 in middle to late Pleistocene strata.

\section{Type $B$}

This is a major fractionation of plagioclase of the hightemperature trend, amphibole or biotite, and rare clinopyroxene. This is related to high-pressure crystallization of waterundersaturated $\left(\mathrm{PH}_{2} \mathrm{O}, 2\right.$ kbar - see above) andesitic magma. High-alumina and moderate alkalies contents may correspond to high-alumina basalt series of an island or continental margin arc. Type B locates Site 769 in late Pliocene to early Pleistocene strata, and Site 767 in Quaternary strata.

\section{Type $C$}

This is a major fractionation of clinopyroxene and plagioclase of the high-temperature trend and subordinate amphibole. This is related to high-pressure crystallization of andesitic magma. High alumina and alkalies contents may correspond to high-K andesite series of an island or continental margin arc. Type C occurs at Sites 768 and 769, in Quaternary strata.

\section{The Second Fractionation Step}

This step, from dacites to rhyolites, concerns titanomagnetites, hornblendes, Mg-biotites, plagioclases (An 45-35), and alkaline feldspars (Or 25-71). Overlapping trends combine removals of clinopyroxenes, titanomagnetites, amphiboles, biotites, plagioclases, and alkaline feldspars. It appears that orthopyroxene is not an intervening phase. OPX only occurs with acidic glasses, except for Sample 62 (124-769B-3H-6, $25-27 \mathrm{~cm}$ ), where coexisting with CPX. In terms of mineral fractionation, high-K rhyolite compositions result in plagioclases + alkaline feldspars removal, whereas low-K rhyolite compositions suggest heavy fractionation of alkaline feldspars. But in many cases, the $\mathrm{K}$-content of acidic glasses is modified by the alteration. Thus, this last fractionation step is poorly defined. 


\section{DISCUSSION}

According to the geochemical and petrological interpretations, the volcaniclastic glasses and minerals are assigned to three magmatic series that occurred throughout the two basins: (1) low-alumina and medium-K hornblende andesitic series, (2) high-alumina and medium- $\mathrm{K}$ basalt series, and (3) high-alumina and high-K pyroxene andesitic to shoshonitic series.

The first question is: What are the sources of these products? The second question will be: What is the correlation between the Leg 124 tephra composition and the geodynamic evolution of the surrounding areas?

\section{Comparison with Land Volcanics}

Various medium- and high- $\mathrm{K}$ calc-alkaline suites are known in neighboring volcanic arcs, located in composite transitional crustal environments with oceanic crusts and continental fragments. Their features are summarized as follows. Timing and apparent tectonic control of volcanism are from Wolfe (1981), Rangin et al. (1990), Bellon and Rangin (this volume), and Bellon (this volume). Petrochemical data are interpreted from Divis (1980), Jesek et al. (1981), Morris et al. (1983), Yuwono (1987), Kudrass et al. (1990), and Bellon and Rangin (this volume).

In the Eastern Philippine magmatic activities occurred from Eocene to early Miocene time in relation with an eastward subduction below the Philippine arc (Eastern Philippines), and since Pliocene, in relation with the westward subduction in the Philippine trench. Lavas consist of pyroxene-hornblende andesites suites of low-alumina and medium-K composition (series A), and also of high-alumina and medium-K basalts (series B).

In the western Philippine (from Zambales to central Mindanao), various volcanic activities are related to complex subductions along the Manila-Sulu trenches, South China Sea and Sulu Sea since the late Miocene. Successive volcanic arcs were accreted (Rangin et al., in press). Some lavas may have low-Al and medium-K composition (series A), but most of the compositions are high-Al and medium- $\mathrm{K}$ or high- $\mathrm{K}$, and differentiation trends belong to series $\mathrm{B}$ and $\mathrm{C}$ defined above. High-K andesites with shoshonitic affinities (series C) seem to locate in transverse structures such as Verde Passage Zone, south of Manila, and central Mindanao.

The South Philippine volcanic zone (South Mindanao and Sangihe arcs) is related to subduction of the Molucca Sea since the late Miocene (Pubellier et al., in press) and of the Celebes Sea in the Cotobato Trench since the early Quaternary. Hornblende andesites are predominent in the northern sector of this zone (series A). High-Al olivine basalts and pyroxene andesites (series B) erupted in the whole zone.

To the south, the Halmahera Island arc fringed the Philippine Plate (southern extension of Philippine arc) from Eocene to Miocene time. Since the Pliocene, volcanism is linked to the Molucca Sea subduction toward the east (Hall et al., 1988). It consists of medium-K basalt through dacite suites of series B. However, this arc was farther east in Pliocene and Miocene times, and too distant from the Leg 124 sites. On the Sulawesi Island, subduction of the Indo-Australian Plate generated a large range of low-K to shoshonite suites since the early Miocene. Similar lavas constituted the Sunda arc since the Eocene to Holocene. This arc is a possible source for the oldest ash deposits cored at Leg 124.

In addition, calc-alkaline volcanic activities occurred along continental strips that separate marginal basins (Rangin, Silver, von Breymann et al., 1990). The Palawan and the Cagayan Ridges were the loci of a medium-K andesite volca- nism related to subductions of the South China Sea passive margin, during the early to middle Miocene (Kudrass et al., 1990). The Sulu Ridge was active in the middle Miocene with subduction of the proto-South China Sea (Holloway, 1982), and since the Pliocene with subduction of the Sulu Sea. Lavas belong to series $\mathrm{B}$ : high- $\mathrm{Al}$ and medium-K andesite suites. At the nearby North Sulawesi arc, new subduction of the Celebes Sea since the late Miocene produced high-Al basalt suite (series B).

To sum up, the various arcs may be petrologically different in some parts (for example, shoshonitic feature of the Negros and central Mindanao volcanoes), but similar magmatic products occur in many distinct areas. There is little information concerning the magmatic evolution of these arcs through time and space (Bellon and Rangin, this volume). Different lava suites may be present at any time in the numerous and successive nearby volcanic arcs. The identity of the sources is inferred from stratigraphic correlations with known durations of volcanism on the arcs. Thus the Leg 124 record allows precise chronology of the explosive pulses in relation with the main volcano-tectonic events (Pubellier et al., this volume).

Considering the Leg 124 ash compositions, their stratigraphic ages, and the relative arc locations, possible sources are given in Table 6 . Sites 767 and 770 may have been supplied by the Sunda, oldest Sulawesi, and Mindanao volcanoes in the Oligocene to early and middle Miocene (acidic volcaniclastites), and by the north Sulawesi, Sangihe, Mindanao, and Sulu volcanoes since the late Miocene (series A and B, basic to acidic tephra). Sites 768 and 769 were obviously supplied by the Cagayan Ridge at the early middle Miocene (hyaloclastite and pyroclastite flows) and by the west Philippine volcanoes, especially by the Negros volcanoes (series C), and the Sulu Archipelago since the mid-Pleistocene.

\section{The Leg 124 Volcanic Record}

Volcanic ash from the Celebes and Sulu Sea Basins provide a good record of the explosive activity of the neighboring arcs since the late Oligocene in the Celebes area and since the early Miocene in the Sulu area. This record is discussed in Pubellier et al., this volume. Chemical and petrological investigations are used to characterize volcanic pulses (Table 6). Notably, successive input of mafic to evolved magmatic products indicate three magmatic sequences: (1) in the early Pliocene, (2) in the late Pliocene to early Pleistocene, and (3) in the middle to late Pleistocene.

This new information provides additional data for palinspastic reconstructions of this western Pacific region (Rangin et al., 1990).

\section{Late Oligocene-Early Miocene Time (Age Groups 10 and 9)}

The Celebes Basin registered two pulses circa 32 and 25 Ma. Poorly characterized andesites and rhyolites may indicate activities of the Sunda arc and of the Sabah old Sulu arc.

\section{Late Early and Middle Miocene Time (Age Groups 8 and 7)}

Diluted volcaniclastic material in the Celebes Basin sediments indicates distant location of sources: Sulawesi to the south and Sulu to the north.

Sulu Sea andesitic through dacitic tuffs originated from the Cagayan volcanic chain circa 18-17 Ma. Hyaloclastites correspond to proximal flows whereas pyroclastites are attributed to more distal deposition of explosive tephra. This activity ended when the Cagayan and Palawan ridges collided with the Philippine Plate (Rangin, 1989; Rangin et al., 1990; Rangin and Pubellier, 1990). In the early late Miocene, circa $10 \mathrm{Ma}$, dacite to evolved rhyolite activity is registered in the Sulu Sea Basin. 
Table 4. Chemical analyses and structural formulas of minerals.

\begin{tabular}{|c|c|c|c|c|c|c|c|c|c|c|c|c|c|c|c|}
\hline \multicolumn{16}{|c|}{ Table $4-\mathrm{I}-\mathrm{A}$} \\
\hline & 5 & 16 & $5^{\prime}$ & 8 & 11 & 24 & 22 & $24^{\prime}$ & 21 & $16^{\prime}$ & 13 & 25 & 27 & $16^{\prime \prime}$ & 25 \\
\hline $\mathrm{SiO}_{2}$ & 49.35 & 49.51 & 54.36 & 56.11 & 57.76 & 59.47 & 62.88 & 64.14 & 65.32 & 65.91 & 65.71 & 66.93 & 65.50 & 65.27 & 64.23 \\
\hline $\mathrm{Al}_{2} \mathrm{O}_{3}$ & 30.27 & 28.88 & 27.23 & 26.38 & 25.05 & 23.71 & 22.39 & 20.29 & 19.75 & 19.06 & 18.20 & 17.72 & 17.18 & 16.85 & 16.94 \\
\hline $\mathrm{Fe}_{2} \mathrm{O}_{3}$ & 0.59 & 0.95 & 0.73 & 0.68 & 0.57 & 0.87 & 0.66 & 0.12 & 0.23 & 0.26 & 0.53 & 0.19 & 0.36 & 0.07 & 0.00 \\
\hline $\mathrm{CaO}^{-}$ & 16.45 & 14.69 & 12.05 & 10.89 & 8.34 & 7.66 & 4.71 & 3.03 & 2.54 & 2.56 & 0.83 & 0.33 & 0.13 & 0.00 & 0.00 \\
\hline $\mathrm{Na}_{2} \mathrm{O}$ & 1.85 & 3.16 & 4.36 & 5.16 & 5.89 & 6.72 & 8.04 & 9.96 & 10.36 & 7.24 & 6.21 & 6.31 & 3.27 & 1.09 & 0.27 \\
\hline $\mathrm{K}_{2} \mathrm{O}$ & 0.07 & 0.23 & 0.23 & 0.28 & 0.53 & 0.54 & 1.63 & 1.15 & 0.82 & 4.18 & 6.94 & 7.81 & 11.60 & 14.64 & 16.59 \\
\hline Total & 98.58 & 97.42 & 98.96 & 99.50 & 98.14 & 98.97 & 100.31 & 98.69 & 99.02 & 99.21 & 98.42 & 99.29 & 98.04 & 97.92 & 98.03 \\
\hline $\mathrm{Si}$ & 2.291 & 2.329 & 2.486 & 2.543 & 2.644 & 2.689 & 2.795 & 2.884 & 2.917 & 2.958 & 3.005 & 3.027 & 3.037 & 3.057 & 3.035 \\
\hline $\mathrm{Al}$ & 1.656 & 1.601 & 1.468 & 1.409 & 1.351 & 1.264 & 1.173 & 1.075 & 1.040 & 1.008 & 0.981 & 0.945 & 0.939 & 0.930 & 0.944 \\
\hline $\mathrm{Fe}^{3+}$ & 0.021 & 0.034 & 0.025 & 0.024 & 0.018 & 0.030 & 0.022 & 0.004 & 0.008 & 0.009 & 0.016 & 0.006 & 0.013 & 0.002 & 0.000 \\
\hline $\mathrm{Ca}$ & 0.818 & 0.740 & 0.590 & 0.529 & 0.409 & 0.371 & 0.224 & 0.146 & 0.121 & 0.123 & 0.041 & 0.016 & 0.006 & 0.000 & 0.000 \\
\hline $\mathrm{Na}$ & 0.167 & 0.288 & 0.387 & 0.454 & 0.523 & 0.589 & 0.693 & 0.868 & 0.897 & 0.630 & 0.551 & 0.553 & 0.294 & 0.099 & 0.025 \\
\hline K & 0.004 & 0.014 & 0.013 & 0.016 & 0.031 & 0.031 & 0.093 & 0.066 & 0.047 & 0.240 & 0.406 & 0.452 & 0.688 & 0.877 & 1.002 \\
\hline An & 82.74 & 71.03 & 59.62 & 52.97 & 42.48 & 37.43 & 22.21 & 13.51 & 11.41 & 12.40 & 4.08 & 1.56 & 0.66 & 0.00 & 0.00 \\
\hline $\mathrm{Ab}$ & 16.84 & 27.65 & 39.03 & 45.4 & 54.30 & 59.42 & 68.62 & 80.37 & 84.20 & 63.45 & 55.23 & 54.20 & 29.75 & 10.15 & 2.40 \\
\hline Or & 0.42 & 1.32 & 1.35 & 1.62 & 3.22 & 3.15 & 9.17 & 6.12 & 4.39 & 24.15 & 40.69 & 44.24 & 69.59 & 89.58 & 97.60 \\
\hline \multicolumn{16}{|c|}{ Table 4-I - B } \\
\hline & 32 & 34 & 47 & 37 & 32 & 44 & 40 & 51 & 29 & 42 & $47^{\prime}$ & 31 & & & \\
\hline $\mathrm{SiO}_{2}$ & 48.52 & 50.05 & 53.34 & 54.82 & 59.16 & 60.22 & 65.44 & 66.88 & 66.67 & 64.42 & 65.72 & 65.96 & & & \\
\hline $\mathrm{Al}_{2} \mathrm{O}_{3}$ & 30.49 & 29.55 & 28.63 & 26.96 & 23.94 & 24.09 & 21.36 & 18.99 & 18.78 & 18.49 & 18.60 & 17.07 & & & \\
\hline $\mathrm{Fe}_{2} \mathrm{O}_{3}$ & 0.74 & 1.19 & 0.88 & 0.17 & 0.52 & 0.72 & 0.18 & 0.23 & 0.12 & 0.53 & 0.00 & 0.10 & & & \\
\hline $\mathrm{CaO}$ & 17.20 & 15.22 & 12.11 & 12.02 & 8.51 & 6.93 & 3.14 & 1.53 & 1.63 & 1.56 & 0.92 & 0.00 & & & \\
\hline $\mathrm{Na}_{2} \mathrm{O}$ & 1.45 & 2.85 & 4.30 & 5.47 & 6.75 & 7.12 & 9.11 & 10.70 & 7.86 & 3.81 & 3.56 & 2.39 & & & \\
\hline $\mathrm{K}_{2} \mathrm{O}$ & 0.01 & 0.15 & 0.09 & 0.11 & 0.17 & 0.71 & 1.52 & 0.10 & 4.10 & 9.18 & 9.41 & 13.44 & & & \\
\hline Total & 98.41 & 99.01 & 99.35 & 99.55 & 99.05 & 99.79 & 100.75 & 98.43 & 99.16 & 97.99 & 98.21 & 98.96 & & & \\
\hline $\mathrm{Si}$ & 2.262 & 2.316 & 2.432 & 2.496 & 2.673 & 2.695 & 2.873 & 2.979 & 2.984 & 2.974 & 3.008 & 3.049 & & & \\
\hline $\mathrm{Al}$ & 1.675 & 1.612 & 1.538 & 1.447 & 1.275 & 1.271 & 1.105 & 0.997 & 0.991 & 1.007 & 1.003 & 0.930 & & & \\
\hline $\mathrm{Fe}^{3+}$ & 0.029 & 0.046 & 0.030 & 0.006 & 0.020 & 0.024 & 0.006 & 0.008 & 0.005 & 0.020 & 0.000 & 0.004 & & & \\
\hline $\mathrm{Ca}$ & 0.859 & 0.755 & 0.592 & 0.586 & 0.412 & 0.333 & 0.148 & 0.073 & 0.078 & 0.077 & 0.045 & 0.000 & & & \\
\hline $\mathrm{Na}$ & 0.131 & 0.256 & 0.380 & 0.483 & 0.591 & 0.618 & 0.775 & 0.924 & 0.682 & 0.341 & 0.316 & 0.214 & & & \\
\hline K & 0.000 & 0.009 & 0.005 & 0.006 & 0.010 & 0.040 & 0.085 & 0.006 & 0.234 & 0.541 & 0.551 & 0.793 & & & \\
\hline An & 86.73 & 74.05 & 60.56 & 54.51 & 40.70 & 33.57 & 14.66 & 7.30 & 7.85 & 8.05 & 4.94 & 0.00 & & & \\
\hline $\mathrm{Ab}$ & 13.23 & 25.09 & 38.91 & 44.89 & 58.35 & 62.35 & 76.89 & 92.12 & 68.59 & 35.56 & 34.66 & 21.26 & & & \\
\hline Or & 0.04 & 0.86 & 0.53 & 0.60 & 0.95 & 4.08 & 8.45 & 0.58 & 23.56 & 56.39 & 60.40 & 78.74 & & & \\
\hline \multicolumn{16}{|c|}{ Table 4 - I - C } \\
\hline Sample & 63 & 64 & 62 & 59 & $62^{\prime}$ & 67 & $62^{\prime \prime}$ & 68 & & & & & & & \\
\hline $\mathrm{SiO}_{2}$ & 48.20 & 50.99 & 51.74 & 53.84 & 56.60 & 59.89 & 61.87 & 66.37 & & & & & & & \\
\hline $\mathrm{Al}_{2} \mathrm{O}_{3}$ & 30.60 & 28.80 & 28.20 & 27.63 & 25.98 & 22.80 & 22.23 & 17.62 & & & & & & & \\
\hline $\mathrm{Fe}_{2} \mathrm{O}_{3}$ & 0.94 & 0.91 & 0.98 & 0.98 & 0.52 & 0.38 & 0.35 & 0.38 & & & & & & & \\
\hline $\mathrm{CaO}$ & 16.23 & 14.16 & 13.87 & 11.45 & 9.87 & 6.35 & 5.42 & 0.22 & & & & & & & \\
\hline $\mathrm{Na}_{2} \mathrm{O}$ & 1.81 & 3.11 & 3.52 & 4.85 & 5.80 & 7.54 & 7.80 & 4.34 & & & & & & & \\
\hline $\mathrm{K}_{2} \mathrm{O}$ & 0.06 & 0.11 & 0.27 & 0.43 & 0.31 & 0.91 & 1.28 & 10.34 & & & & & & & \\
\hline Total & 97.84 & 98.08 & 98.58 & 99.18 & 99.08 & 97.87 & 98.95 & 99.27 & & & & & & & \\
\hline $\mathrm{Si}$ & 2.258 & 2.371 & 2.395 & 2.461 & 2.569 & 2.730 & 2.785 & 3.026 & & & & & & & \\
\hline $\mathrm{Al}$ & 1.689 & 1.578 & 1.539 & 1.488 & 1.390 & 1.225 & 1.179 & 0.947 & & & & & & & \\
\hline $\mathrm{Fe}^{3+}$ & 0.037 & 0.035 & 0.038 & 0.038 & 0.020 & 0.015 & 0.013 & 0.015 & & & & & & & \\
\hline $\mathrm{Ca}$ & 0.815 & 0.705 & 0.688 & 0.561 & 0.480 & 0.310 & 0.261 & 0.011 & & & & & & & \\
\hline $\mathrm{Na}$ & 0.164 & 0.281 & 0.316 & 0.430 & 0.510 & 0.666 & 0.681 & 0.383 & & & & & & & \\
\hline K & 0.004 & 0.067 & 0.016 & 0.025 & 0.018 & 0.053 & 0.074 & 0.601 & & & & & & & \\
\hline An & 82.90 & 71.03 & 67.46 & 55.22 & 47.61 & 30.14 & 25.74 & 1.07 & & & & & & & \\
\hline $\mathrm{Ab}$ & 16.71 & 28.29 & 30.96 & 42.31 & 50.60 & 64.74 & 67.02 & 38.53 & & & & & & & \\
\hline Or & 0.39 & 0.67 & 1.58 & 2.47 & 1.79 & 5.12 & 7.24 & 60.41 & & & & & & & \\
\hline \multicolumn{16}{|c|}{ Table 4 - II } \\
\hline & 5 & 6 & 8 & 11 & 13 & $13^{\prime}$ & 16 & 34 & 36 & & & & & & \\
\hline $\mathrm{SiO}_{2}$ & & 49.90 & 55.68 & 52.22 & 52.69 & 47.29 & 53.62 & 52.51 & & & & & & & \\
\hline $\mathrm{Al}_{2} \mathrm{O}_{3}$ & 3.42 & 3.96 & 0.66 & 3.31 & 2.12 & 7.52 & 1.68 & 3.29 & 2.20 & & & & & & \\
\hline $\mathrm{Fe}_{2} \mathrm{O}_{3}$ & 0.00 & 1.13 & 0.00 & 0.74 & 0.88 & 4.01 & 0.00 & 0.28 & 0.00 & & & & & & \\
\hline $\mathrm{FeO}$ & 9.03 & 9.82 & 18.38 & 17.35 & 7.73 & 3.70 & 9.06 & 7.74 & 8.65 & & & & & & \\
\hline $\mathrm{MnO}$ & 0.24 & 0.19 & 1.04 & 0.69 & 0.51 & 0.08 & 0.56 & 0.24 & 0.26 & & & & & & \\
\hline $\mathrm{MgO}$ & 15.09 & 13.45 & 23.39 & 23.86 & 15.44 & 13.51 & 15.35 & 14.74 & 14.49 & & & & & & \\
\hline $\mathrm{CaO}$ & 19.83 & 19.07 & 1.34 & 1.51 & 20.59 & 21.99 & 19.93 & 21.20 & 20.69 & & & & & & \\
\hline $\mathrm{Na}_{2} \mathrm{O}$ & 0.30 & 0.47 & 0.05 & 0.03 & 0.27 & 0.21 & 0.17 & 0.40 & 0.05 & & & & & & \\
\hline $\mathrm{TiO}_{2}$ & 0.46 & 1.01 & 0.17 & 0.21 & 0.42 & 0.50 & 0.36 & 0.52 & 0.65 & & & & & & \\
\hline Total & 100.98 & 99.00 & 100.71 & 99.92 & 100.65 & 98.81 & 100.73 & 100.93 & 101.35 & & & & & & \\
\hline $\mathrm{Si}$ & 1.930 & 1.884 & 2.039 & 1.914 & 1.940 & 1.771 & 1.977 & 1.925 & 2.000 & & & & & & \\
\hline Al IV & 0.070 & 0.116 & 0.000 & 0.086 & 0.060 & 0.229 & 0.023 & 0.075 & 0.000 & & & & & & \\
\hline Al VI & 0.078 & 0.060 & 0.028 & 0.056 & 0.032 & 0.103 & 0.050 & 0.067 & 0.095 & & & & & & \\
\hline $\mathrm{Fe}^{3+}$ & 0.000 & 0.032 & 0.000 & 0.020 & 0.024 & 0.113 & 0.000 & 0.008 & 0.000 & & & & & & \\
\hline $\mathrm{Fe}^{2+}$ & 0.288 & 0.310 & 0.562 & 0.531 & 0.238 & 0.116 & 0.279 & 0.237 & 0.265 & & & & & & \\
\hline
\end{tabular}


Table 4 (continued).

\begin{tabular}{lccccccccc}
\hline $\mathrm{Mn}$ & 0.007 & 0.006 & 0.032 & 0.021 & 0.016 & 0.002 & 0.017 & 0.007 & 0.008 \\
$\mathrm{Mg}$ & 0.825 & 0.757 & 1.277 & 1.303 & 0.847 & 0.754 & 0.844 & 0.805 & 0.795 \\
$\mathrm{Ca}$ & 0.779 & 0.771 & 0.052 & 0.059 & 0.812 & 0.882 & 0.787 & 0.833 & 0.815 \\
$\mathrm{Na}$ & 0.021 & 0.034 & 0.003 & 0.002 & 0.019 & 0.015 & 0.012 & 0.028 & 0.004 \\
$\mathrm{Ti}$ & 0.013 & 0.029 & 0.005 & 0.006 & 0.012 & 0.014 & 0.010 & 0.014 & 0.018 \\
Wo & 35.90 & 34.22 & 2.06 & 1.50 & 38.17 & 33.36 & 38.18 & 38.58 & 39.73 \\
$\mathrm{En}$ & 41.38 & 37.86 & 65.71 & 65.17 & 42.37 & 37.71 & 42.57 & 40.28 & 41.03 \\
$\mathrm{Fs}$ & 14.24 & 15.78 & 30.57 & 27.62 & 12.68 & 5.91 & 14.95 & 12.22 & 14.13 \\
$\mathrm{Jd}$ & 2.14 & 0.22 & 0.36 & 0.00 & 0.00 & 0.00 & 1.23 & 2.06 & 0.37 \\
$\mathrm{Ac}$ & 0.00 & 3.22 & 0.00 & 0.21 & 1.93 & 1.52 & 0.00 & 0.79 & 0.00 \\
$\mathrm{Ca}-T s c h$ & 5.07 & 5.83 & 0.80 & 3.09 & 3.19 & 10.30 & 2.06 & 4.64 & 2.88 \\
Fa-Tsch & 0.00 & 0.00 & 0.00 & 1.83 & 0.50 & 9.78 & 0.00 & 0.00 & 0.00 \\
Ti-Tsch & 1.27 & 2.87 & 0.48 & 0.58 & 1.16 & 1.41 & 1.01 & 1.43 & 1.86
\end{tabular}

Table 4 - II

\begin{tabular}{|c|c|c|c|c|c|c|c|c|}
\hline & 50 & 54 & $54^{\prime}$ & 62 & $62^{\prime}$ & 64 & 65 & 70 \\
\hline $\mathrm{SiO}_{2}$ & 54.12 & 51.35 & 51.94 & 55.08 & 51.76 & 53.27 & 55.15 & 52.87 \\
\hline $\mathrm{Al}_{2} \mathrm{O}_{3}$ & 1.32 & 2.87 & 3.41 & 2.26 & 2.72 & 2.33 & 0.73 & 1.95 \\
\hline $\mathrm{Fe}_{2} \mathrm{O}_{3}$ & 0.10 & 1.00 & 1.84 & 0.00 & 1.89 & 0.00 & 0.00 & 0.00 \\
\hline $\mathrm{FeO}$ & 19.92 & 8.61 & 2.70 & 13.02 & 8.87 & 8.12 & 14.51 & 9.53 \\
\hline $\mathrm{MnO}$ & 0.97 & 0.28 & 0.18 & 0.68 & 0.57 & 0.50 & 0.66 & 0.28 \\
\hline $\mathrm{MgO}$ & 23.54 & 14.91 & 16.47 & 27.61 & 14.95 & 14.96 & 26.74 & 15.19 \\
\hline $\mathrm{CaO}$ & 1.44 & 19.60 & 22.89 & 0.64 & 19.28 & 19.86 & 1.06 & 20.67 \\
\hline $\mathrm{Na}_{2} \mathrm{O}$ & 0.03 & 0.29 & 0.18 & 0.00 & 0.35 & 0.32 & 0.06 & 0.15 \\
\hline $\mathrm{TiO}_{2}$ & 0.12 & 0.56 & 0.31 & 0.40 & 0.57 & 0.33 & 0.14 & 0.14 \\
\hline Total & 101.57 & 99.47 & 99.92 & 99.89 & 100.97 & 99.69 & 99.05 & 100.78 \\
\hline Si & 1.968 & 1.918 & 1.899 & 1.974 & 1.911 & 1.979 & 2.007 & 1.948 \\
\hline Al IV & 0.032 & 0.082 & 0.101 & 0.026 & 0.089 & 0.021 & 0.000 & 0.062 \\
\hline Al VI & 0.025 & 0.044 & 0.045 & 0.069 & 0.030 & 0.081 & 0.031 & 0.022 \\
\hline $\mathrm{Fe}^{3+}$ & 0.003 & 0.028 & 0.050 & 0.000 & 0.053 & 0.000 & 0.000 & 0.000 \\
\hline $\mathrm{Fe}^{2+}$ & 0.605 & 0.268 & 0.082 & 0.389 & 0.274 & 0.252 & 0.441 & 0.293 \\
\hline $\mathrm{Mn}$ & 0.030 & 0.009 & 0.006 & 0.021 & 0.018 & 0.016 & 0.020 & 0.009 \\
\hline $\mathrm{Mg}$ & 1.276 & 0.830 & 0.898 & 1.485 & 0.823 & 0.829 & 1.451 & 0.834 \\
\hline $\mathrm{Ca}$ & 0.056 & 0.784 & 0.897 & 0.025 & 0.762 & 0.790 & 0.041 & 0.816 \\
\hline $\mathrm{Na}$ & 0.002 & 0.021 & 0.013 & 0.000 & 0.025 & 0.023 & 0.004 & 0.011 \\
\hline $\mathrm{Ti}$ & 0.003 & 0.016 & 0.008 & 0.011 & 0.016 & 0.009 & 0.004 & 0.004 \\
\hline Wo & 1.37 & 35.86 & 39.94 & 1.10 & 34.48 & 38.07 & 1.41 & 38.74 \\
\hline En & 63.81 & 41.50 & 44.80 & 75.4 & 41. & 2 & 73.45 & 41.50 \\
\hline Fs & 31.74 & 13.86 & 4.39 & 20.8 & 14. & 13.5 & 23.25 & 15.02 \\
\hline Jd & 0.00 & 0.00 & 0.00 & 0.00 & 0.0 & 2.3 & 0.43 & 1.07 \\
\hline Ac & 0.21 & 2.10 & 1.27 & 0.00 & 2.51 & 0.00 & 0.00 & 0.00 \\
\hline Ca-Tsch & 2.46 & 4.39 & 4.22 & 2.68 & 2.96 & 3.07 & 0.98 & 3.29 \\
\hline Fa-Tsch & 0.08 & 0.71 & 4.52 & 0.00 & 2.76 & 0.00 & 0.00 & 0.00 \\
\hline \multirow[t]{3}{*}{ Ti-Tsch } & 0.33 & 1.57 & 0.85 & 1.10 & 1.58 & 0.93 & 0.39 & 0.39 \\
\hline & & & & & & & \multicolumn{2}{|c|}{ Table 4 - II } \\
\hline & 1 & 3 & 8 & 13 & 14 & 19 & 33 & 37 \\
\hline $\mathrm{SiO}_{2}$ & 46.58 & 54.37 & 47.71 & 44.27 & 44.14 & 45.01 & 49.41 & 43.11 \\
\hline $\mathrm{Al}_{2} \mathrm{O}_{3}$ & 8.82 & 6.09 & 7.79 & 10.94 & 12.41 & 10.02 & 7.01 & 13.47 \\
\hline $\mathrm{Fe}_{2} \mathrm{O}_{3}$ & 8.64 & 6.40 & 3.84 & 5.63 & 7.04 & 5.65 & 7.01 & 5.14 \\
\hline $\mathrm{FeO}$ & 4.31 & 4.16 & 8.30 & 7.34 & 4.67 & 9.19 & 6.75 & 3.88 \\
\hline $\mathrm{MnO}$ & 0.33 & 0.37 & 0.38 & 0.29 & 0.25 & 0.59 & 0.38 & 0.24 \\
\hline $\mathrm{MgO}$ & 14.50 & 15.79 & 14.44 & 13.70 & 14.85 & 12.44 & 14.92 & 15.58 \\
\hline $\mathrm{CaO}$ & 10.62 & 9.67 & 10.99 & 11.53 & 11.6 & 10. & 10.96 & 12.07 \\
\hline $\mathrm{Na}_{2} \mathrm{O}$ & 1.66 & 1.10 & 2.06 & 2.18 & 2.36 & 2.04 & 1.79 & 2.51 \\
\hline $\mathrm{K}_{2} \mathrm{O}$ & 0.29 & 0.27 & 0.37 & 0.53 & 0.54 & 0.82 & 0.38 & 0.45 \\
\hline $\mathrm{TiO}_{2}$ & 1.38 & 0.59 & 2.12 & 1.20 & 1.47 & 2.18 & 1.24 & 1.30 \\
\hline Total & 97.13 & 98.81 & 97.99 & 97.61 & 99.39 & 98.78 & 99.85 & 97.75 \\
\hline $\mathrm{Si}$ & 6.727 & 7.509 & 6.880 & 6.459 & 6.276 & 6.543 & 6.975 & 6.193 \\
\hline $\mathrm{Al}$ & 1.501 & 0.991 & 1.324 & 1.881 & 2.080 & 1.717 & 1.166 & 2.281 \\
\hline $\mathrm{Fe}^{3+}$ & 0.939 & 0.665 & 0.417 & 0.619 & 0.753 & 0.618 & 0.745 & 0.555 \\
\hline $\mathrm{Fe}^{2+}$ & 0.521 & 0.481 & 1.000 & 0.896 & 0.556 & & 0.797 & 0.466 \\
\hline $\mathrm{Mn}$ & 0.040 & 0.043 & 0.046 & 0.036 & 0.030 & 0.073 & 0.045 & 0.029 \\
\hline $\mathrm{Mg}$ & 3.121 & 3.250 & 3.103 & 2.979 & 3.147 & 2.695 & 3.139 & 3.335 \\
\hline $\mathrm{Ca}$ & 1.643 & 1.431 & 1.698 & 1.802 & 1.775 & 1.688 & 1.658 & 1.858 \\
\hline $\mathrm{Na}$ & 0.465 & 0.295 & 0.576 & 0.617 & 0.651 & 0.575 & 0.490 & 0.699 \\
\hline K & 0.054 & 0.048 & 0.068 & 0.099 & 0.098 & 0.152 & 0.069 & 0.083 \\
\hline $\mathrm{Ti}$ & 0.150 & 0.061 & 0.230 & 0.132 & 0.157 & 0.238 & 0.132 & 0.140 \\
\hline $\begin{array}{l}\mathrm{Mg} / \mathrm{Mg} \\
+\mathrm{Fe}^{2+}\end{array}$ & 84.76 & 86.12 & 74.78 & 76.18 & 84.31 & 69.37 & 78.84 & 87.08 \\
\hline
\end{tabular}

Table 4 - III

$\begin{array}{lrrrrrrr} & 41 & 45 & 48 & 48^{\prime} & 64 & 68 & 70 \\ \mathrm{SiO}_{2} & 47.90 & 48.82 & 48.35 & 45.35 & 43.98 & 49.77 & 50.37 \\ \mathrm{Al}_{2} \mathrm{O}_{3} & 8.33 & 7.76 & 7.45 & 10.83 & 10.35 & 6.59 & 5.59 \\ \mathrm{Fe}_{2} \mathrm{O}_{3} & 14.25 & 10.57 & 10.36 & 8.42 & 6.17 & 8.59 & 9.99\end{array}$


Table 4 (continued).

\begin{tabular}{|c|c|c|c|c|c|c|c|}
\hline $\mathrm{FeO}$ & 0.01 & 2.43 & 3.42 & 2.12 & 12.52 & 6.42 & 2.80 \\
\hline $\mathrm{MnO}$ & 0.37 & 0.33 & 0.27 & 0.21 & 0.63 & 0.16 & 0.35 \\
\hline $\mathrm{MgO}$ & 14.85 & 15.53 & 15.26 & 15.86 & 10.25 & 14.14 & 16.40 \\
\hline $\mathrm{CaO}$ & 9.36 & 10.42 & 10.57 & 10.90 & 11.30 & 10.42 & 10.78 \\
\hline $\mathrm{Na}_{2} \mathrm{O}$ & 1.49 & 1.47 & 1.54 & 2.24 & 1.48 & 1.40 & 1.23 \\
\hline $\mathrm{K}_{2} \mathrm{O}$ & 0.15 & 0.25 & 0.14 & 0.36 & 1.37 & 0.09 & 0.10 \\
\hline $\mathrm{TiO}_{2}$ & 1.03 & 0.97 & 1.54 & 1.59 & 1.05 & 1.22 & 1.14 \\
\hline Total & 97.74 & 98.55 & 98.90 & 97.87 & 98.48 & 98.80 & 98.75 \\
\hline $\mathrm{Si}$ & 6.793 & 6.890 & 6.838 & 6.462 & 6.505 & 7.071 & 7.086 \\
\hline $\mathrm{Al}$ & 1.392 & 1.291 & 1.242 & 1.819 & 1.804 & 1.104 & 0.927 \\
\hline $\mathrm{Fe}^{3+}$ & 1.520 & 1.123 & 1.102 & 0.903 & 0.686 & 0.918 & 1.057 \\
\hline $\mathrm{Fe}^{2+}$ & 0.001 & 0.287 & 0.405 & 0.252 & 1.549 & 0.763 & 0.329 \\
\hline $\mathrm{Mn}$ & 0.044 & 0.039 & 0.032 & 0.025 & 0.079 & 0.019 & 0.042 \\
\hline $\mathrm{Mg}$ & 3.139 & 3.267 & 3.216 & 3.368 & 2.259 & 2.994 & 3.438 \\
\hline $\mathrm{Ca}$ & 1.422 & 1.576 & 1.602 & 1.664 & 1.791 & 1.568 & 1.625 \\
\hline $\mathrm{Na}$ & 0.410 & 0.402 & 0.422 & 0.619 & 0.424 & 0.386 & 0.335 \\
\hline K & 0.027 & 0.045 & 0.025 & 0.067 & 0.259 & 0.016 & 0.018 \\
\hline $\mathrm{Ti}$ & 0.110 & 0.103 & 0.164 & 0.170 & 0.117 & 0.130 & 0.121 \\
\hline $\begin{array}{l}\mathrm{Mg} / \mathrm{Mg} \\
+\mathrm{Fe}^{27}\end{array}$ & 98.57 & 90.92 & 88.04 & 92.39 & 58.12 & 79.28 & 90.25 \\
\hline
\end{tabular}

\begin{tabular}{|c|c|c|c|c|c|c|c|c|c|c|c|c|}
\hline & & & & & & & & ble $4-1 v$ & & & & \\
\hline & 8 & 25 & $25^{\prime}$ & 28 & 33 & 36 & 40 & $40^{\prime}$ & 43 & 50 & 62 & 68 \\
\hline $\mathrm{SiO}_{2}$ & 0.58 & 0.45 & 0.26 & 0.85 & 0.52 & 1.10 & 0.74 & 0.84 & 0.00 & 0.00 & 2.93 & 1.08 \\
\hline $\mathrm{Al}_{2} \mathrm{O}_{3}$ & 1.48 & 2.02 & 0.68 & 2.06 & 2.00 & 3.32 & 2.05 & 0.71 & 0.28 & 0.18 & 4.76 & 2.32 \\
\hline $\mathrm{Fe}_{2} \mathrm{O}_{3}$ & 48.39 & 48.05 & 47.62 & 36.61 & 56.80 & 49.80 & 57.58 & 47.64 & 21.36 & 10.94 & 48.80 & 50.78 \\
\hline $\mathrm{FeO}$ & 38.39 & 35.58 & 23.54 & 40.78 & 33.18 & 33.71 & 32.94 & 22.68 & 29.61 & 38.99 & 31.88 & 35.50 \\
\hline $\mathrm{MnO}$ & 0.55 & 0.94 & 0.41 & 0.85 & 0.61 & 0.59 & 0.37 & 0.42 & 1.12 & 0.89 & 0.30 & 0.63 \\
\hline $\mathrm{MgO}$ & 0.49 & 2.03 & 0.36 & 2.68 & 1.65 & 2.34 & 1.33 & 0.66 & 3.92 & 1.56 & 4.05 & 1.74 \\
\hline $\mathrm{CaO}$ & 0.06 & 0.00 & 0.00 & 0.06 & 0.13 & 0.17 & 0.03 & 0.00 & 0.00 & 0.00 & 0.21 & 0.11 \\
\hline $\mathrm{TiO}_{2}$ & 9.46 & 9.52 & 26.81 & 15.85 & 5.44 & 7.64 & 4.64 & 26.46 & 41.74 & 47.32 & 7.84 & 8.21 \\
\hline Total & 99.40 & 98.59 & 99.67 & 99.74 & 100.33 & 98.67 & 99.68 & 99.41 & 98.03 & 99.88 & 100.77 & 100.37 \\
\hline $\mathrm{Si}$ & 0.175 & 0.135 & 0.013 & 0.249 & 0.154 & 0.326 & 0.221 & 0.043 & 0.000 & 0.000 & 0.820 & 0.318 \\
\hline & 0.527 & 0.715 & 0.041 & 0.710 & 0.700 & 1.158 & 0.123 & & 0.017 & 0.011 & 1.570 & 0.804 \\
\hline $\mathrm{Fe}^{3+}$ & 10.994 & 10.854 & 1.848 & 8.059 & 12.696 & 11.090 & 12.964 & 1.841 & 0.811 & 0.413 & 10.279 & 11.233 \\
\hline $\mathrm{Fe}^{2+}$ & 9.693 & 8.932 & 1.015 & 9.977 & 8.242 & 8.343 & 8.242 & 0.974 & 1.249 & 1.637 & 7.462 & 8.727 \\
\hline $\mathrm{Mn}$ & 0.141 & 0.239 & 0.018 & 0.211 & 0.153 & 0.148 & 0.094 & 0.018 & 0.048 & 0.038 & 0.071 & 0.157 \\
\hline $\mathrm{Mg}$ & 0.221 & 0.908 & 0.027 & 1.168 & 0.730 & 1.032 & 0.593 & 0.051 & 0.295 & 0.117 & 1.689 & 0.762 \\
\hline $\mathrm{Ca}$ & 0.019 & 0.000 & 0.000 & 0.019 & 0.041 & 0.054 & 0.010 & 0.000 & 0.000 & 0.000 & 0.063 & 0.035 \\
\hline $\mathrm{Ti}$ & 2.148 & 2.149 & 1.040 & 3.487 & 1.215 & 1.700 & 1.044 & 1.022 & 1.583 & 1.786 & 1.650 & 1.815 \\
\hline
\end{tabular}

$\begin{array}{lrrr} & 17 & 31 & 37 \\ \mathrm{SiO}_{2} & 37.89 & 39.11 & 39.09 \\ \mathrm{Al}_{2} \mathrm{O}_{3} & 13.07 & 12.23 & 13.15 \\ \mathrm{FeO} & 17.50 & 15.56 & 12.20 \\ \mathrm{MnO} & 0.26 & 0.28 & 0.04 \\ \mathrm{MgO} & 13.79 & 14.75 & 16.87 \\ \mathrm{CaO} & 0.00 & 0.00 & 0.30 \\ \mathrm{Na}_{2} \mathrm{O} & 0.31 & 0.44 & 0.84 \\ \mathrm{~K}_{2} \mathrm{O} & 8.90 & 9.27 & 7.60 \\ \mathrm{TiO}_{2} & 3.91 & 3.37 & 3.21 \\ \mathrm{H}_{2} \mathrm{O} & 3.71 & 3.37 & 4.02 \\ \mathrm{Total} & 99.34 & 99.00 & 97.31 \\ \mathrm{Si} & 5.703 & 5.873 & 5.825 \\ \mathrm{Al} & 2.319 & 2.165 & 2.309 \\ \mathrm{Fe} & 2.203 & 1.954 & 1.521 \\ \mathrm{Mn} & 0.033 & 0.036 & 0.005 \\ \mathrm{Mg} & 3.093 & 3.301 & 3.747 \\ \mathrm{Ca} & 0.000 & 0.000 & 0.048 \\ \mathrm{Na} & 0.090 & 0.128 & 0.241 \\ \mathrm{~K} & 1.713 & 1.780 & 1.444 \\ \mathrm{Ti} & 0.443 & 0.381 & 0.360 \\ \mathrm{H}_{2} \mathrm{O} & 2.000 & 2.000 & 2.000 \\ \mathrm{Mg} / \mathrm{Mg} & 58.04 & 62.39 & 71.05 \\ +\mathrm{Fe} & & & \\ +\mathrm{Mn} & & & \end{array}$

5

$\mathrm{SiO}_{2} \quad 39.92$

$\mathrm{FeO} \quad 18.73$

$\mathrm{MnO} \quad 0.13$

$\mathrm{MgO} \quad 41.06$

$\mathrm{CaO} \quad 0.15$

$\begin{array}{rr}\mathrm{TiO}_{2} & 0.00 \\ & 99.99\end{array}$ 
Table 4 (continued).

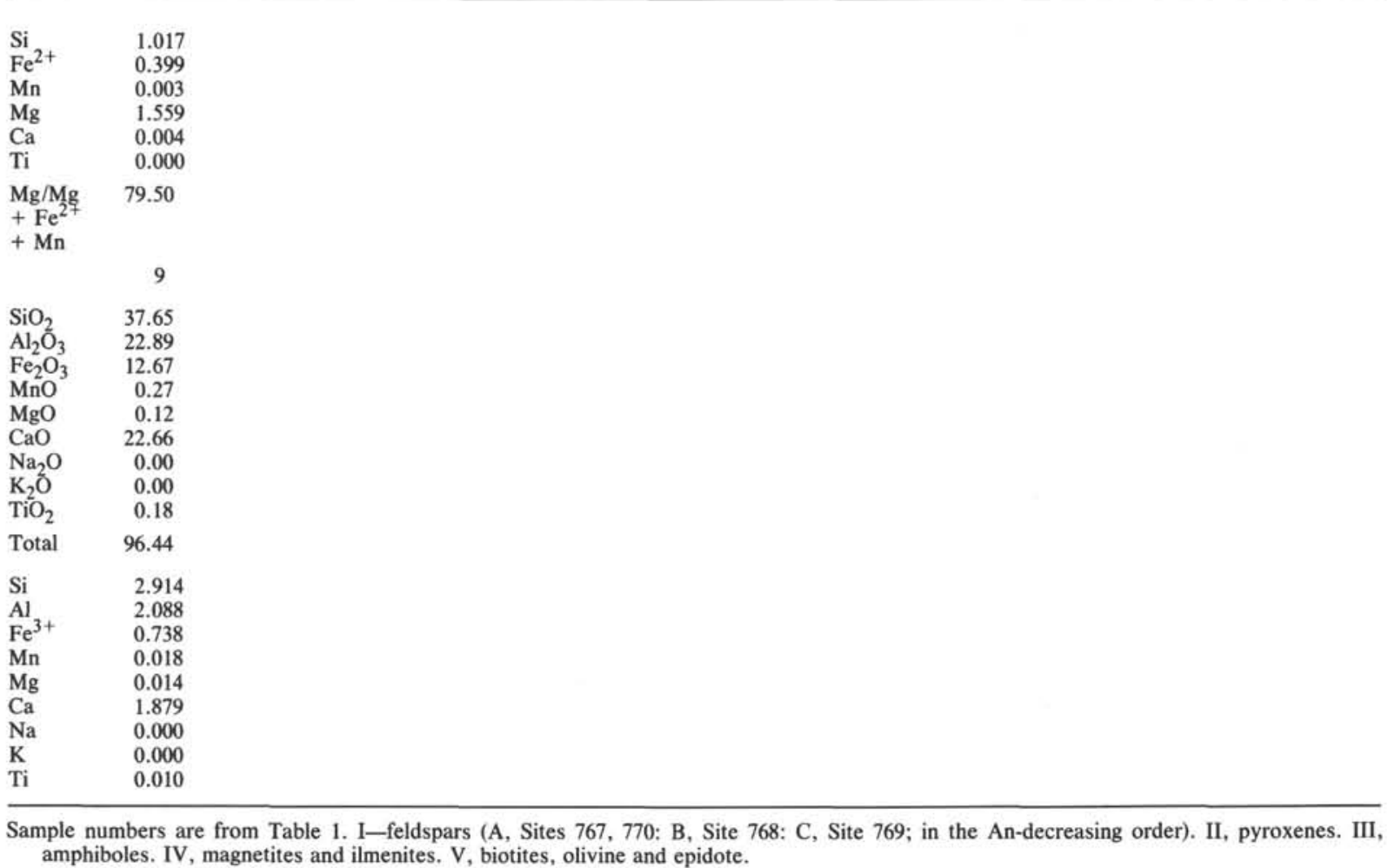
amphiboles. IV, magnetites and ilmenites. V, biotites, olivine and epidote.

At that time the Philippine arc (carried by the Philippine Plate) collided with the Eurasian margin.

\section{Late Miocene and Early Pliocene Time (Age Groups 6 and 5)}

In the late Miocene, renewal of volcanic activity is indicated at the two basins since 8-7.5 Ma. Magmatic compositions are poorly defined. A better record is registered at all the sites in the early Pliocene (magmatic sequence I, Table 6). This is in agreement with the new tectonic setting that followed the major compression event in the late late Miocene: left-lateral movement of the Philippine fault, reversal of subduction orientation beneath Sulu Archipelago, subduction of the Philippine Sea, and beginning closure of the Molucca Sea (Divis, 1980; Cardwell and Isacks, 1981; Mukasa et al., 1987; Rangin, Silver, von Breymann, et al., 1990). Celebes Sea ash layers give evidence of newly born volcanoes of the Sangihe immature arc (type A fractionation trend). Sulu Sea sites denote activity of the new Sulu-Zamboanga arc and of the Negros volcanoes.

\section{Late Pliocene-Middle Pleistocene Time (Age Groups 4 and 3)}

The apparent absence of volcanic ash between 3.5-2.5 Ma may be explained, partly, by dissolution of vitric glasses (Desprairies et al., this volume), or by the period of absence of activity between the Sangihe subduction and the Cotobato trench in Mindanao (Pubellier et al., in press). We may assume that tectonic constraints are limited to strike-slip movements in the central Philippines. However, a new volcano-tectonic pulse occurred at $2.5 \mathrm{Ma}$ (magmatic sequence II, Table 6). In the Celebes Sea Basin, from late Pliocene to mid-Pleistocene time, the two magmatic compositions of ashes denote an activity of the Sangihe arc or the North Sulawesi arc and a contribution from the Mindanao and the Sulu volcanoes. In the Sulu Sea Basin, ash beds registered moderate activity from the Sulu-Zamboanga arc and from the Negros volcanoes.

Middle Pleistocene-Holocene Time (Age Groups 2 and 1)

The last important magmatic phase occurred in the late Pleistocene (sequence III, Table 6), with renewal of all the present south Philippine arcs: Cotobato and Sulu (Sites 767 and 770), and Sulu and Negros (Sites 768 and 769). This phase is still continuing, to a lesser extent, giving mafic products from nearby volcanic chains and episodic acidic explosive tephra dispersed in sea basins.

\section{CONCLUSION}

The volcaniclastic material recovered during Leg 124 in the Celebes and Sulu Sea Basins gives a good record of the nearby volcanic arc activities since the Oligocene. The material originated from Vulcanian to Plinian explosive eruptions and was deposited as fallout ash layers in the sedimentary pile of the two basins and as pyroclastite flows in Miocene strata of the Sulu Basin.

Mineralogical and geochemical studies of 70 samples lead us to distinguish three magmatic series: (1) low-alumina and medium- $\mathrm{K}$ andesitic series, (2) high-alumina and medium-K basalt series, and (3) high-alumina and high- $\mathrm{K}$ andesitic to shoshonitic series. Some volcanic suites occurred at various places and times in the numerous surrounding volcanic arcs and ridges.

In a first step, the identity of the sources is inferred from stratigraphic correlations with known durations of volcanism on the arcs. Thus the Leg 124 record allows a precise chronology of the volcanic pulses. In a second step, interesting information is deducted from the volcanological and petrological investigations. The Sulu Sea hyaloclastite and pyroclastite tuffs originated 

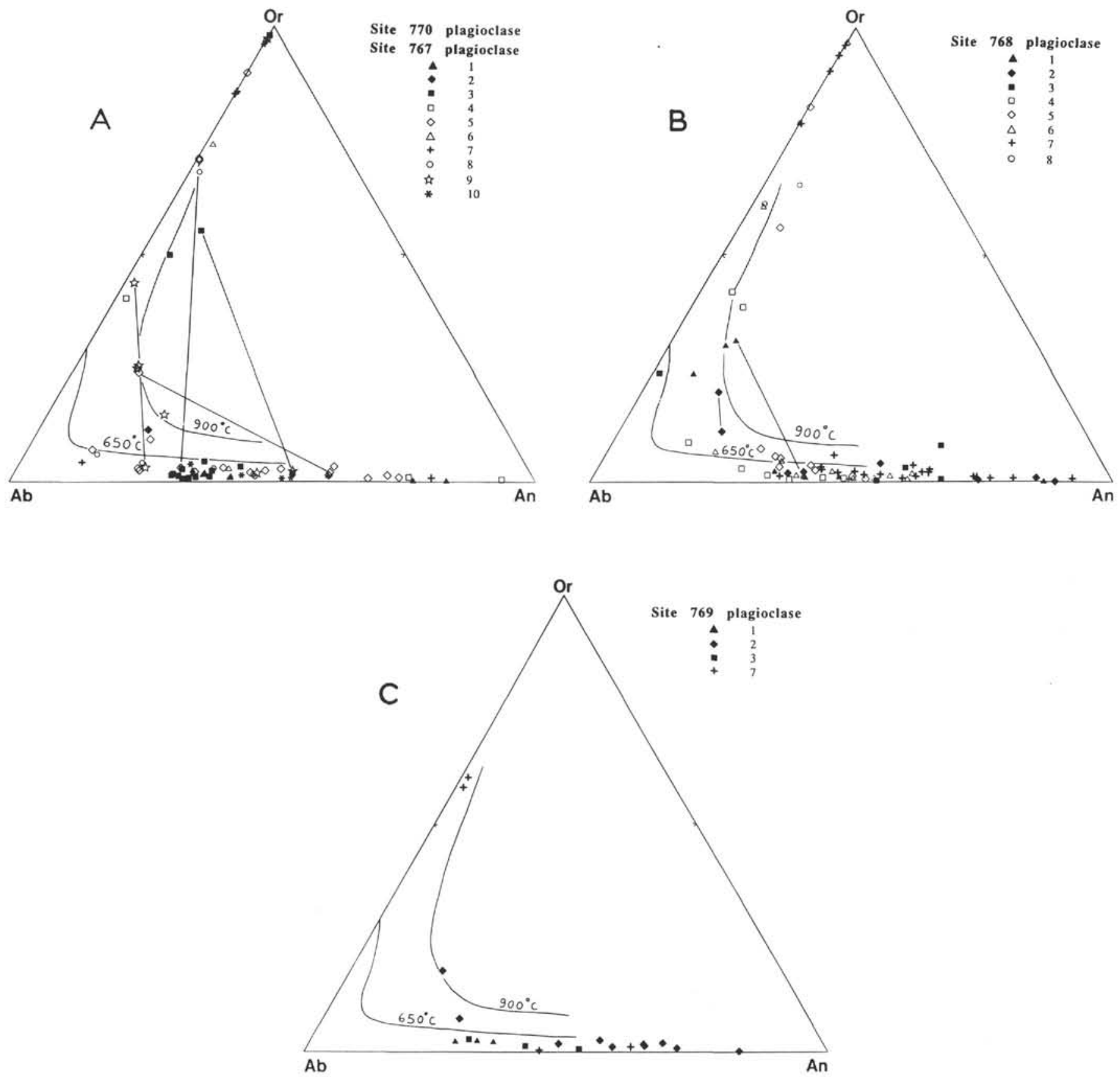

Figure 5. An-Ab-Or diagram of the feldspars. A. Sites 767, 770. B. Site 768. C. Site 769. 1 to 10 , symbols for the age groups. Tie-lines join coexisting feldspars. Limits of feldspar solid solutions at $\mathrm{P}_{2} \mathrm{O}=1 \mathrm{kbar}$ for $900^{\circ} \mathrm{C}$ and $650^{\circ} \mathrm{C}$ according to Seck (1971).

from the Cagayan volcanic chain in the early middle Miocene. After that, the Cagayan ridge activity stopped. Low-alumina hornblende-andesite lava suites are recorded when a new arc becomes active (Sangihe-north-Sulawesi). A more complex magmatic activity (three sequences) is distinguished in the PliocenePleistocene. This may correspond to successive stages in the new tectonic setting that followed the major compression event in the late late Miocene.

\section{ACKNOWLEDGMENT}

We greatly appreciate conspicuous and highly pertinent suggestions and questions from the two reviewers, James Natland and Richard Fisher, and from Claude Rangin. We thank Michel Tallet for his skillful participation in the microprobe analyses. This research was supported by a CNRS grant (ODP-France).

\section{REFERENCES}

Andersen, D. J., and Lindsley, D. H., 1988. Internally consistent solution models for $\mathrm{Fe}-\mathrm{Mg}-\mathrm{Mn}-\mathrm{Ti}$ oxides: $\mathrm{Fe}-\mathrm{Ti}$ oxides. $\mathrm{Am}$. Mineral., 73:714-726.

Barth, T.F.W., 1962. Feldspar solid solutions. Chem. Erde, 22:31.

Cardwell, R. K., and Isacks, B. L., 1981. A review of the configuration of the Eastern Indonesian and Philippine islands. In The Geology and Tectonics of Eastern Indonesia. Geol. Res. Develop. Centre, Spec. Publ., 2:31-47. 
Divis, A. F., 1980. The petrology and tectonics of recent volcanism in the Central Philippine islands. In Hayes, D. E. (Ed.), The Tectonic and Geologic Evolution of Southeast Asian Seas and Islands. Am. Geophys. Union., Geophys. Monogr., 27:127-144.

Fisher, R. V., 1961. Proposed classification of volcaniclastic sediments and rocks. Geol. Soc. Am. Bull., 72:1409-1414.

Fisher, R. V., 1966. Rocks composed of volcanic fragments and their classification. Earth-Sci. Rev., 1:287-298.

Fisher, R. V., and Schmincke, H.-U., 1984. Pyroclastic Rocks: New York (Springer-Verlag).

Hall, R., Audley-Charles, M. G., Banner, F. T., Hidayat, S., and Tobing, S. L., 1988. Late Paleogene-Ouaternary geology of Halmahera, Eastern Indonesia: initiation of a volcanic island arc. J. Geol. Soc. London, 145:577-590.

Hammarstrom, J. M., and Zen, E.-A., 1986. Aluminium in hornblende: an empirical igneous geobarometer. Am. Mineral., 71:1297-1313.

Hollister, L. S., Grimssom, G. C., Peters, E. K., Stowell, H. H., and Sisson, V. B., 1987. Confirmation of the empirical correlation of $\mathrm{Al}$ in hornblende with pressure of solidification of calc-alkaline plutons. Am. Mineral., 72:231-239.

Holloway, N. H., 1982. The stratigraphy and tectonic relationship of Reed Bank, North Palawan, and Mindoro to the Asian Mainland and its significance in the evolution of the South China Sea. AAPG Bull., 66:1357-1383.

Huang, T. C., 1980. A volcanic sedimentation model: implication of processes and responses of deep-sea ashes. Mar. Geol., 38:103122.

Jezek, P. A., Whitford, D. J., and Gill, J. B., 1981. Geochemistry of recent lavas from the Sangihe-Sulawesi arc, Indonesia. In The Geology and Tectonics of Eastern Indonesia. Geol. Res. Dev. Cent. Spec. Publ., 2:383-389.

Kudo, A. M., and Weill, D. F., 1970. An igneous plagioclase thermometer. Contrib. Mineral. Petrol., 25:52-65.

Kudrass, H. R., Müller, P., Kreuzer, H., and Weiss, W., 1990. Volcanic rocks and tertiary carbonates dredged from the Cagayan Ridge and the Southwest Sulu Sea, Philippines. In Rangin, C., Silver, E. A., von Breymann, M. T., et al., Proc. ODP, Init. Repts., 124: College Station, TX (Ocean Drilling Program), 93100.

Mathez, E. A., 1973. Refinement of the Kudo-Weill plagioclase thermometer and its application to basaltic rocks. Contrib. Mineral. Petrol., 41:61-72.

Morris, J. D., Jezek, P. A., Hart, S. R., and Gill, J. B., 1983. The Halmahera island arc, Molucca Sea collision zone, Indonesia: a geochemical survey. In Hayes, D. E. (Ed.), The Tectonic and Geologic Evolution of Southeast Asian Seas and Islands (Pt. 2). Am. Geophys. Union, Geophys. Monogr., 27:373-387.

Mukasa, S. B., McCabe, R., and Gill, J. B., 1987. Pb-isotopic compositions of volcanic rocks in the West and East Philippine island arcs: presence of the Dupal isotopic anomaly. Earth Planet. Sci. Lett., 84:153-164.

Pouclet, A., Cadet, J.-P., Fujioka, K., and Bourgois, J., 1985. Ash layers from Deep Sea Drilling Project Leg 84: Middle America Trench transect. In von Huene, R., Aubouin, J., et al., Init.
Repts. DSDP, 84: Washington (U.S. Govt. Printing Office), 609-618.

Pouclet, A., Cambray, H., Cadet, J.-P., Bourgois, J., and De Wever, P., 1990. Volcanic ash from Leg 112 off Peru. In Suess, E., von Huene, R., et al., Proc. ODP, Sci. Results, 112: College Station, TX (Ocean Drilling Program), 465-480.

Pubellier, M., Quebral, R., Rangin, C., Deffontaines, B., Muller, C., Butterlin, J., and Manzano, J., in press. The Mindanao collision zone: a soft collision event within a continuous Neogene strike slip setting. Spec. Iss. J. South East Asian Earth Sci.

Rangin, C., 1989. The Sulu Sea, a back-arc basin setting within a Neogene collision zone. Tectonophysics, 161:119-141.

Rangin, C., Jolivet, L., Pubellier, M., and Tethys Pacific Working Group, 1990. A simple model for the tectonic evolution of Southeast Asia and Indonesia regions for the past $43 \mathrm{Ma}$. Bull. Soc. Geol. Fr., 6:887-905.

Rangin, C., Pubellier, M., 1990. Subduction and accretion of oceanic fragments along the Eurasian margin: southern Japan-Philippine region. Some constrains for continental growth. In Aubouin, J., and Bourgois, J. (Eds.), Tectonics of Circum Pacific Continental Margins, (V.S.P International Publ.), 139-144.

Rangin, C., Silver, E., von Breymann, M. T., et al., 1990. Proc. ODP, Init. Repts., 124: College Station, TX (Ocean Drilling Program).

Rangin, C., Stephan, J.-F., Butterlin, J., Bellon, H., Müller. C., and Chorowicz, J., in press. Collision neogène d'arcs volcaniques dans le centre des Philippines. Stratigraphie et structure de la chaîne d'Antique, île de Panay. Bull. Soc. Geol. Fr.

Seck, H. A., 1971. Koexistierende alkali-feldspate und plagioklase im system $\mathrm{NaAlSi}_{3} \mathrm{O}_{8}$ - $\mathrm{KAlSi}_{3} \mathrm{O}_{8}-\mathrm{CaAl}_{2} \mathrm{Si}_{2} \mathrm{O}_{8}-\mathrm{H}_{2} \mathrm{O}$ bei temperaturen von $650^{\circ} \mathrm{C}$ bis $900^{\circ} \mathrm{C}$. Neues Jahrb. Mineral. Abh., 115:315-395.

Stormer, J. C., Jr., 1983. The effects of recalculation on estimates of temperature and oxygen fugacity from analyses of multicomponent iron-titanium oxides. Am. Mineral., 68:586-594.

Wells, P.R.A., 1977. Pyroxene thermometry in simple and complex systems. Contrib. Mineral. Petrol., 62:129-139.

Wolfe, J. A., 1981. Philippine geochronology. J. Soc. Geol. Phil., $35: 1-30$.

Wones, D. R., and Eugster, H. P., 1965. Stability of biotite: experiment, theory and application. Am. Mineral., 50:1228-1272.

Wright, T. L., and Doherty, P. C., 1970. A linear programming and least squares computer method for solving petrologic mixing problems. Geol. Soc. Am. Bull., 81:1995-2008.

Yuwono, Y. S., 1987. Contribution à l'étude du volcanisme potassique de l'Indonéie. Exemples du sud-ouest de Sulawesi et du volcan Muria (Java) [Ph.D. dissert.]. Univ. Brest, France.

Date of initial receipt: 19 June 1990

Date of acceptance: 18 February 1991

Ms 124B-144 

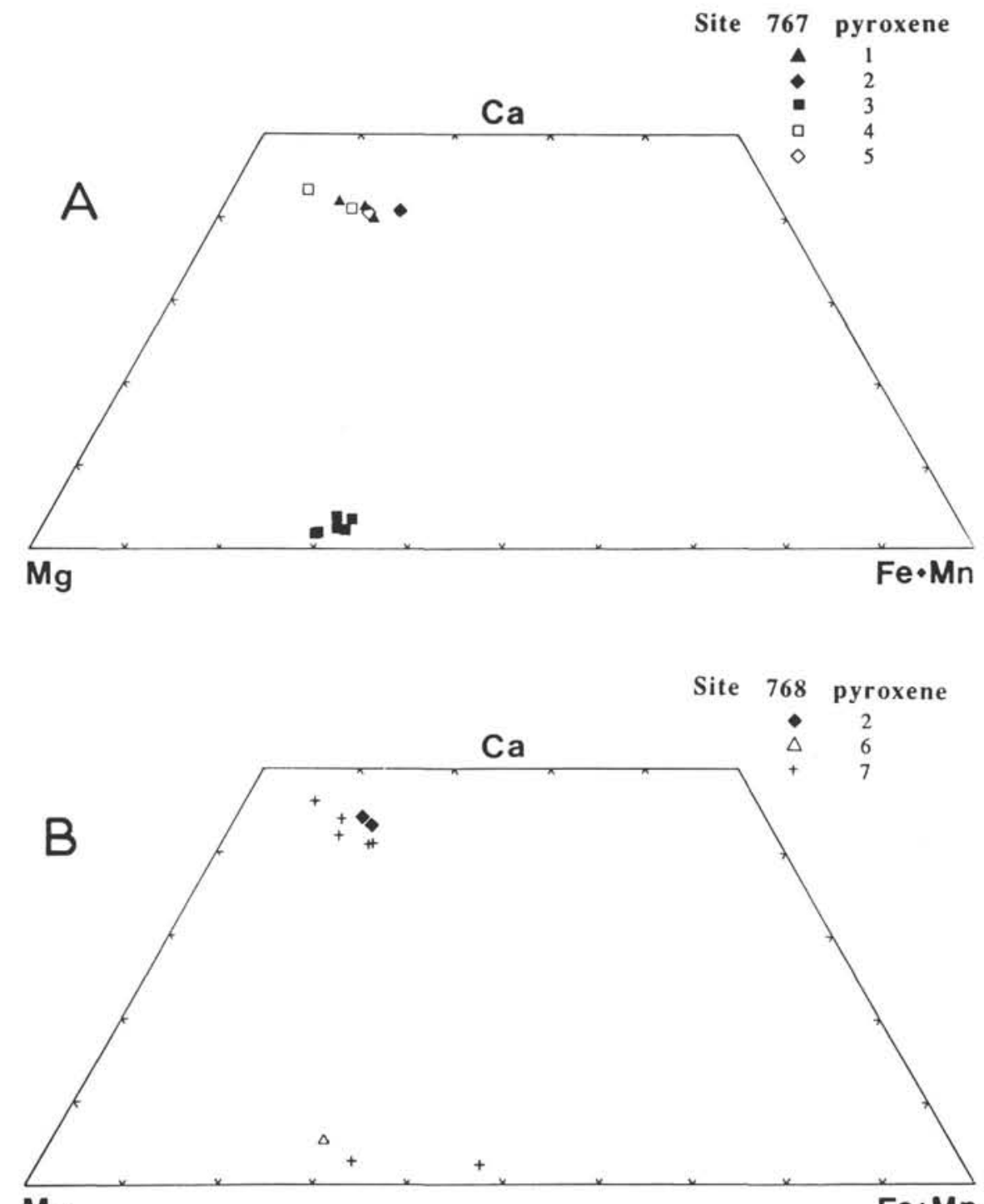

$\mathrm{Mg}$

$\mathrm{Fe} \cdot \mathrm{Mn}$

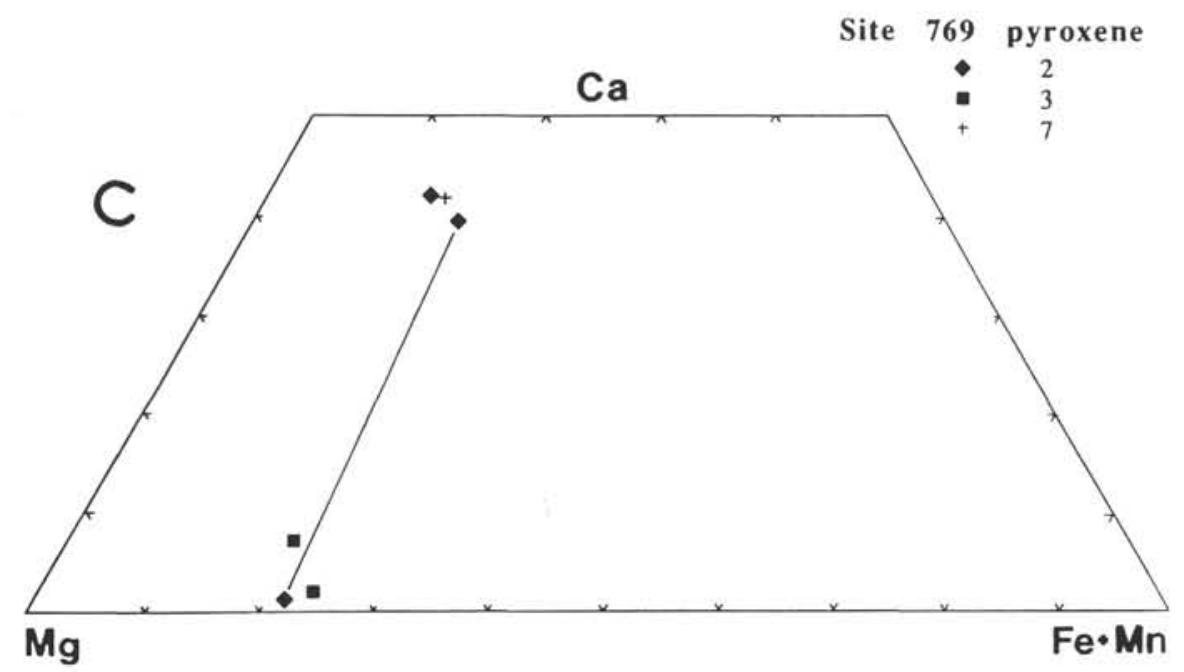

Figure 6. Mg-Ca-Fe + Mn diagram of the pyroxenes. A. Site 767. B. Site 768. C. Site 769. Numbers 1 to 10 show age groups. Tie-lines join coexisting pyroxenes. 

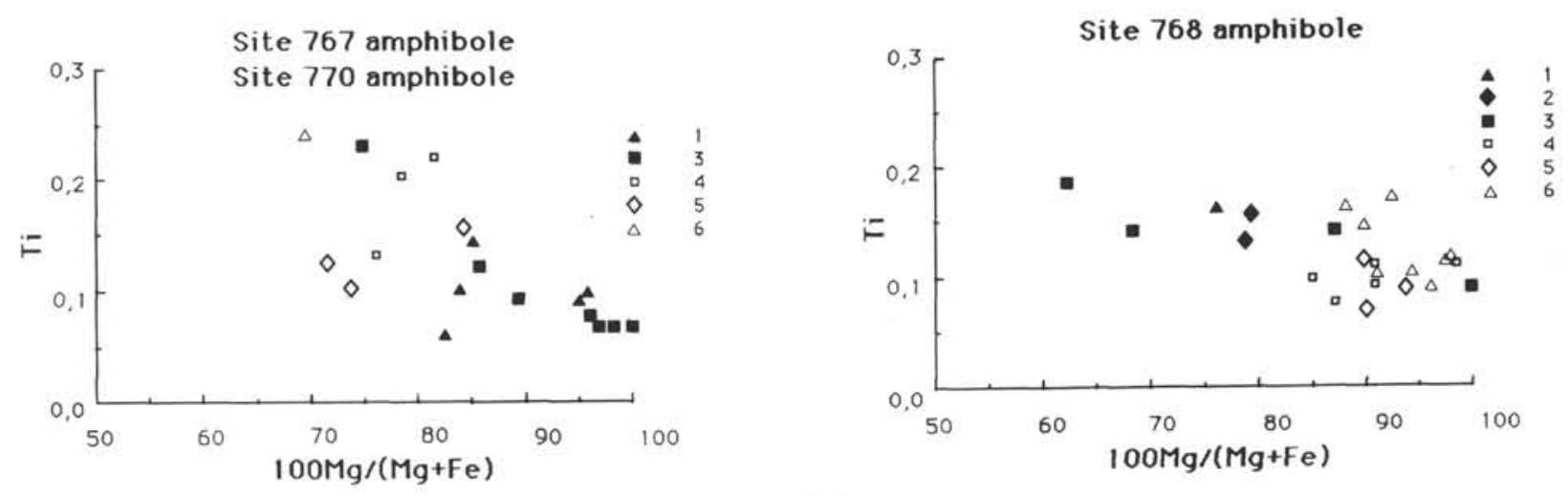

A

B
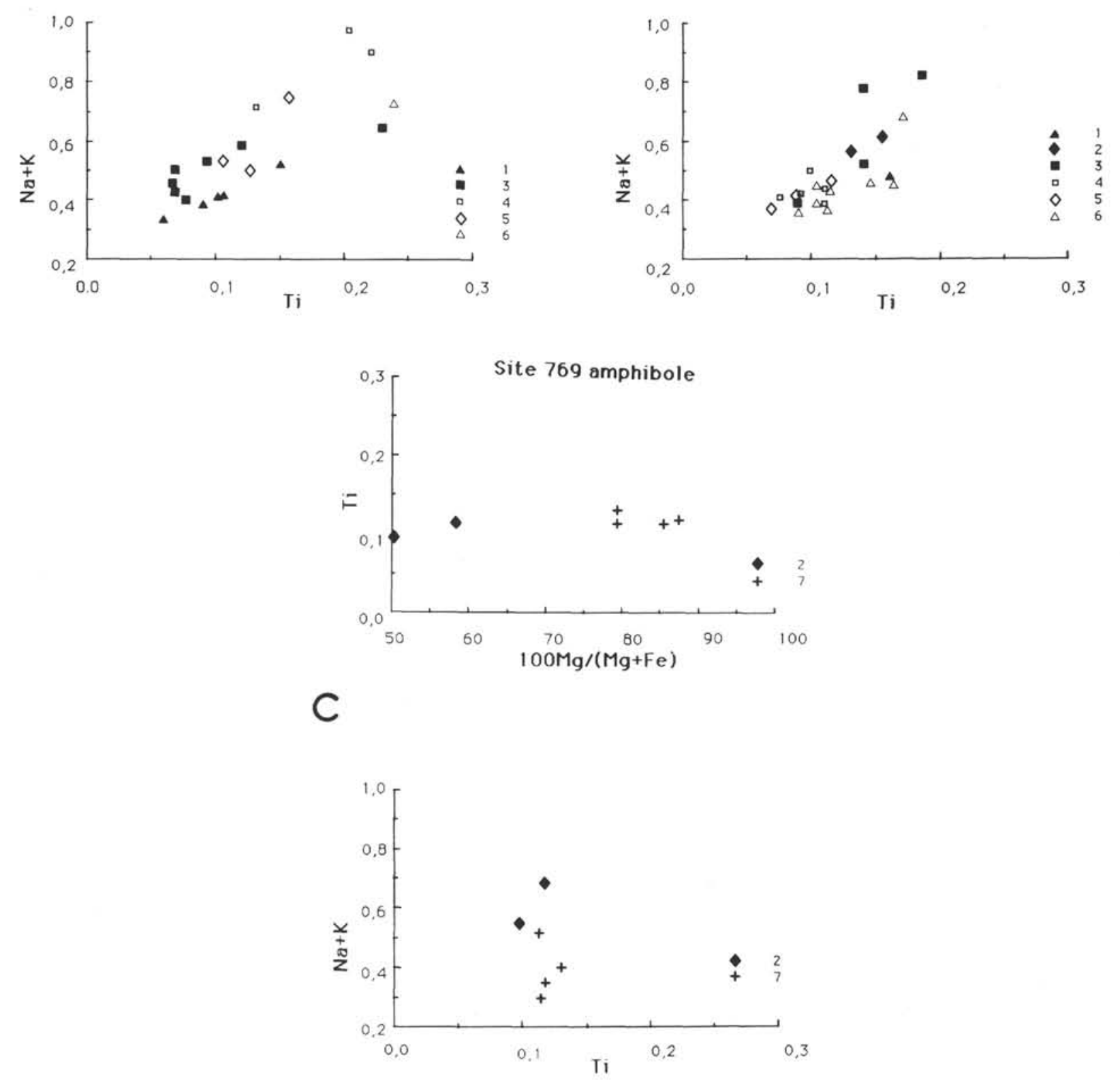

Figure 7. $100 \mathrm{Mg} /(\mathrm{Mg}+\mathrm{Fe})$ vs. Ti and Ti vs. $\mathrm{Na}+\mathrm{K}$ diagrams for amphibole determination. A. Sites 767, 770. B. Site 768. C. Site 769. Numbers 1 to 10 show age groups. 

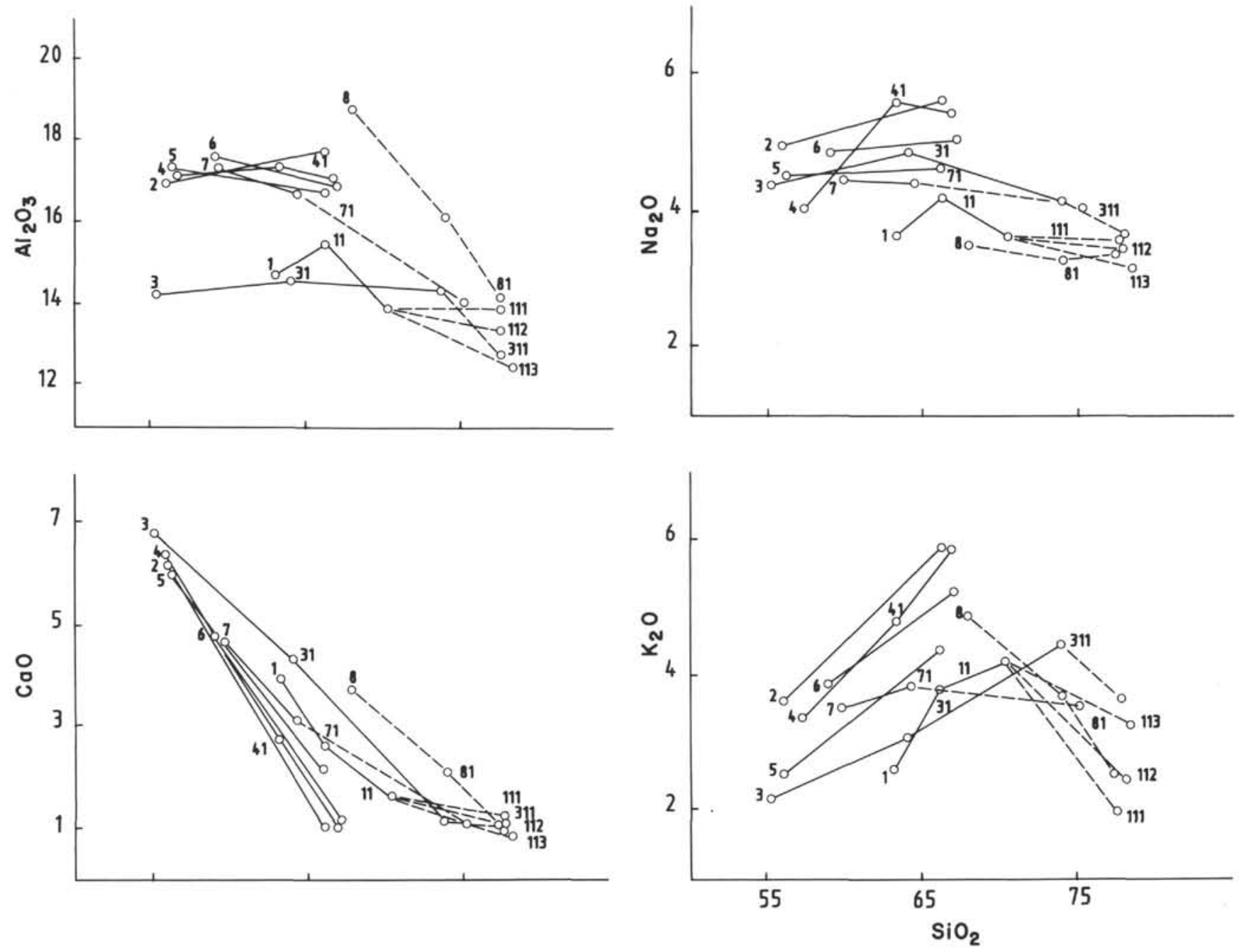

Figure 8. $\mathrm{SiO}_{2}$ vs. $\mathrm{Al}_{2} \mathrm{O}_{3}, \mathrm{CaO}, \mathrm{Na}_{2} \mathrm{O}$, and $\mathrm{K}_{2} \mathrm{O}$ crystallization trends (see text). 
Table 5. Selected least-square mass balance calculations.

\begin{tabular}{|c|c|c|c|c|c|c|c|c|c|c|c|}
\hline Site & Trend & $\begin{array}{l}\text { Sample no. } \\
\text { parent-daug. }\end{array}$ & $\begin{array}{l}\text { Age } \\
\text { group }\end{array}$ & CPX & $\begin{array}{l}\% \mathrm{~F} \\
\mathrm{Mt}\end{array}$ & $\begin{array}{l}\text { actionated } \\
\text { Am }\end{array}$ & $\underset{\mathrm{Bi}}{\text { minerals }^{2}}$ & PI & F & $\begin{array}{l}\text { Residual } \\
\text { squared }\end{array}$ & $\begin{array}{c}\% \\
\text { daughter }\end{array}$ \\
\hline \multirow[t]{5}{*}{767} & 1 & $14.1-14.2$ & 5 & 6.4 & 4.0 & - & - & $5.4(4)$ & - & 0.871 & 84.2 \\
\hline & 11 & $14.2-14.3$ & 5 & - & 2.0 & $4.8(\mathrm{H})$ & - & $10.5(4)$ & $18.5(6)$ & 0.652 & 64.2 \\
\hline & 111 & $14.3-9$ & $5-3$ & - & 4.5 & - & 0.5 & - & $28.9(9)$ & 0.556 & 66.1 \\
\hline & 112 & $14.3-10$ & $5-3$ & - & 4.4 & - & 0.7 & - & $27.3(9)$ & 0.829 & 67.6 \\
\hline & 113 & $14.3-3$ & 5.1 & - & 4.3 & - & 1.2 & $10.5(4)$ & $20.0(9)$ & 0.418 & 41.8 \\
\hline 769 & 2 & $62.1-62.2$ & 2 & 11.3 & 5.2 & $9.0(\mathrm{P})$ & - & $22.5(2)$ & - & 1.800 & 52.0 \\
\hline \multirow[t]{3}{*}{769} & 3 & $64.1-64.2$ & 2 & 1.0 & 4.5 & $24.2(\mathrm{P})$ & - & $7.8(1)$ & - & 0.588 & 62.5 \\
\hline & 31 & $64.2-64.3$ & 2 & 12.4 & 4.6 & - & - & 27.9 (5) & - & 1.166 & 55.1 \\
\hline & 311 & $64.3-61$ & $2-1$ & - & - & - & 2.5 & - & $25.2(8)$ & 0.530 & 53.0 \\
\hline \multirow[t]{2}{*}{768} & 4 & $35.1-35.2$ & 2 & 8.0 & 5.1 & $3.7(\mathrm{P})$ & - & $16.1(1)$ & - & 0.878 & 67.2 \\
\hline & 41 & $35.2-35.3$ & 2 & - & 1.4 & $6.3(\mathrm{P})$ & - & 16.3 (4) & - & 0.174 & 76.0 \\
\hline 769 & 5 & $67.1-67.2$ & 3 & - & 4.6 & 19.9(P) & - & $22.6(2)$ & - & 1.261 & 52.9 \\
\hline 767 & 6 & $6.1-6.2$ & 2 & 0.7 & 3.1 & $11.6(\mathrm{P})$ & - & $19.8(1)$ & - & 0.873 & 64.8 \\
\hline \multirow[t]{3}{*}{769} & 7 & $66.1-66.2$ & 3 & 3.2 & 2.5 & - & 5.8 & $25.0(4)$ & - & 0.547 & 63.5 \\
\hline & 71 & $66.2-65$ & 3 & 2.5 & 2.8 & - & 7.8 & 27.7 (4) & $18.9(7)$ & 0.548 & 40.3 \\
\hline & $71^{\prime}$ & $66.2-65$ & 3 & - & 2.6 & - & 9.7 & 33.7 (4) & $8.1(10)$ & 0.617 & 46.0 \\
\hline \multirow[t]{2}{*}{768} & 8 & $52-53$ & 7 & - & - & - & 1.3 & $20.1(1)$ & $21.1(10)$ & 0.124 & 57.5 \\
\hline & 81 & $53-51$ & 7 & - & - & - & - & $11.5(3)$ & 14.1 (10) & 0.440 & 74.4 \\
\hline
\end{tabular}

Trend and age, see text. Sample no. from Table 2. Mineral analyses from Table 4: H, hornblende; P, pargasite. (1) to (10), feldspar compositions: 1, An60-Or1.4; 2, An50-Or5; 3, An45-Or5; 4, An35-Or4,3; 5, An20-Or10; 6, Or24-An12; 7 , Or41-An5.7; 8, Or45-An5; 9, Or55-An11; 10, Or71-An0.4.

Abbreviations: daug, daughter; $\mathrm{CPX}$, clinopyroxene; Mt, magnetite; $\mathrm{Am}$, amphibole; $\mathrm{Bi}$, biotite; $\mathrm{Pl}$, plagioclase; F, alkaline feldspar.

Table 6. Petrochemical features of the successive volcanic activities according to the recorded tephra, and their possible location.

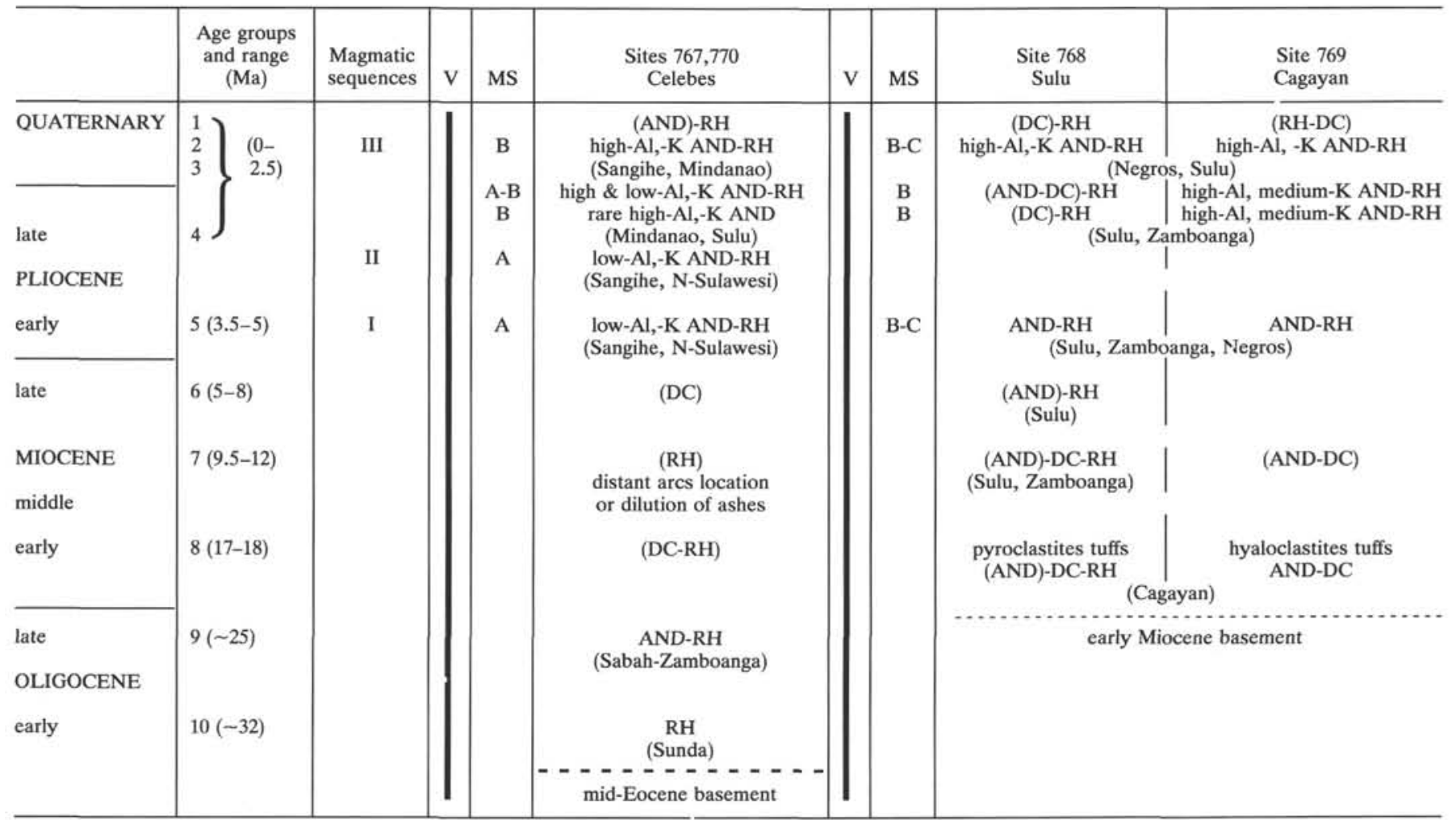

